\title{
High-Temperature Synthesis of CdSe-Based Core/Shell, Core/Shell/Shell, and Core/Graded-Shell Nanoplatelets for Stable and Efficient Narrowband Emitters
}

\author{
Aurelio A. Rossinelli, Henar Rojo, Aniket S. Mule, Marianne Aellen, Ario Cocina, Eva De Leo, Robin \\ Schäublin, David J. Norris
}

Submitted date: 27/06/2019 - Posted date: 28/06/2019

Licence: CC BY-NC-ND 4.0

Citation information: Rossinelli, Aurelio A.; Rojo, Henar; Mule, Aniket S.; Aellen, Marianne; Cocina, Ario; De Leo, Eva; et al. (2019): High-Temperature Synthesis of CdSe-Based Core/Shell, Core/Shell/Shell, and Core/Graded-Shell Nanoplatelets for Stable and Efficient Narrowband Emitters. ChemRxiv. Preprint.

Colloidal semiconductor nanoplatelets exhibit exceptionally narrow photoluminescence spectra. This occurs because samples can be synthesized in which all nanoplatelets share the same atomic-scale thickness. As this dimension sets the emission wavelength, inhomogeneous linewidth broadening due to size variation, which is always present in samples of quasi-spherical nanocrystals (quantum dots), is essentially eliminated. Nanoplatelets thus offer improved, spectrally pure emitters for various applications. Unfortunately, due to their non-equilibrium shape, nanoplatelets also suffer from low photo-, chemical, and thermal stability, which limits their use. Moreover, their poor stability hampers the development of efficient synthesis protocols for adding high-quality protective inorganic shells, which are well known to improve the performance of quantum dots. Herein, we report a general synthesis approach to highly emissive and stable core/shell nanoplatelets with various shell compositions, including CdSe/ZnS, CdSe/CdS/ZnS, CdSe/Cd $\mathrm{Zn}_{1-\mathrm{x}} \mathrm{S}$, and CdSe/ZnSe.

Motivated by previous work on quantum dots, we find that slow, high-temperature growth of shells containing a compositional gradient reduces strain-induced crystal defects and minimizes the emission linewidth while maintaining good surface passivation and nanocrystal uniformity. Indeed, our best core/shell nanoplatelets $\left(\mathrm{CdSe} / \mathrm{Cd}_{x} \mathrm{Zn}_{1-\mathrm{x}} \mathrm{S}\right)$ show photoluminescence quantum yields of $90 \%$ with linewidths as low as $56 \mathrm{meV}$ (19.5 $\mathrm{nm}$ at $655 \mathrm{~nm}$ ). To confirm the high quality of our different core/shell nanoplatelets for a specific application, we demonstrate their use as gain media in low-threshold ring lasers. More generally, the ability of our synthesis protocol to engineer high-quality shells can help further improve nanoplatelets for optoelectronic devices.

File list (2) 


\title{
High-Temperature Synthesis of CdSe-Based
}

\section{Core/Shell, Core/Shell/Shell, and Core/Graded-Shell Nanoplatelets for Stable and Efficient} Narrowband Emitters

\author{
Aurelio A. Rossinelli,,$^{\dagger}$ Henar Rojo, ${ }^{\dagger}$ Aniket S. Mule,$^{\dagger}$ Marianne Aellen, ${ }^{\dagger}$ Ario Cocina ${ }^{\dagger}$ \\ Eva De Leo, ${ }^{\dagger}$ Robin Schäublin ${ }^{\dagger}$ and David J. Norris ${ }^{*}{ }^{\dagger}$ \\ ${ }^{\dagger}$ Optical Materials Engineering Laboratory, Department of Mechanical and Process Engineering, ETH \\ Zurich, 8092 Zurich, Switzerland \\ ¿aboratory of Metal Physics and Technology, Department of Materials, \\ ETH Zurich, 8092 Zurich, Switzerland
}

\begin{abstract}
Colloidal semiconductor nanoplatelets exhibit exceptionally narrow photoluminescence spectra. This occurs because samples can be synthesized in which all nanoplatelets share the same atomic-scale thickness. As this dimension sets the emission wavelength, inhomogeneous linewidth broadening due to size variation, which is always present in samples of quasi-spherical nanocrystals (quantum dots), is essentially eliminated. Nanoplatelets thus offer improved, spectrally pure emitters for various applications. Unfortunately, due to their non-equilibrium shape, nanoplatelets also suffer from low photo-, chemical, and thermal stability, which limits their use. Moreover, their poor stability hampers the development of efficient synthesis protocols for adding high-quality protective inorganic shells, which are well known to improve the performance of quantum dots. Herein, we report a general synthesis approach to highly emissive and stable core/shell nanoplatelets with various shell compositions, including $\mathrm{CdSe} / \mathrm{ZnS}, \mathrm{CdSe} / \mathrm{CdS} / \mathrm{ZnS}, \mathrm{CdSe} / \mathrm{Cd}_{\mathrm{x}} \mathrm{Zn}_{1-\mathrm{x}} \mathrm{S}$, and $\mathrm{CdSe} / \mathrm{ZnSe}$. Motivated by previous work on quantum dots, we find that slow, high-temperature growth of shells containing a compositional gradient reduces strain-induced crystal defects and minimizes the emission linewidth while maintaining good surface passivation and nanocrystal uniformity. Indeed, our best core/shell nanoplatelets $\left(\mathrm{CdSe} / \mathrm{Cd}_{x} \mathrm{Zn}_{1-\mathrm{x}} \mathrm{S}\right)$ show photoluminescence quantum yields of $90 \%$ with linewidths as low as $56 \mathrm{meV}(19.5 \mathrm{~nm}$ at $655 \mathrm{~nm})$. To confirm the high quality of our different core/shell nanoplatelets for a specific application, we demonstrate their use as gain media in low-threshold ring lasers. More generally, the ability of our synthesis protocol to engineer high-quality shells can help further improve nanoplatelets for optoelectronic devices.
\end{abstract}




\section{INTRODUCTION}

Photoluminescence, the process by which a material absorbs and re-emits light, is critical for many photonic and optoelectronic devices. Consequently, highly luminescent semiconductor nanocrystals have been developed for applications such as solid-state lighting, displays, and lasers. ${ }^{1-5}$ When a nanocrystal absorbs a photon, an electron-hole pair (or exciton) is created whose energy is affected by the nanocrystal boundary. Electron-hole recombination can then lead to the emission of a photon with a wavelength tunable via nanocrystal size and shape. State-of-the-art nanocrystals have been designed to exploit this effect and exhibit efficient luminescence in different wavelength ranges. ${ }^{6}$ Arguably, the most studied nanocrystals have been those with a quasi-spherical shape, particles known as colloidal quantum dots (QDs). However, even after several decades of development, the best QD samples still exhibit a size distribution. This leads to spectral broadening in the absorption and emission characteristics, affecting performance in nanocrystal-based devices.

This broadening can be significantly reduced in a newer class of semiconductor nanocrystals known as nanoplatelets (NPLs). These particles have a thin rectangular shape with a uniform thickness of only a few atomic monolayers and lateral sizes of $\sim 10$ to 100 s of nanometers. ${ }^{7-8}$ In the case of CdSe NPLs, the thickness is sufficiently small that photoexcited electron-hole pairs are strongly confined in this one dimension. ${ }^{9}$ The optical properties are then governed by this thickness. If the lateral sizes are above $10 \mathrm{~nm}$, variations in these other two dimensions have negligible impact. ${ }^{10}$ Also, because samples can be prepared in which all NPLs have the exact same thickness (e.g., 4 monolayers), NPLs can provide narrower absorption and emission lines than QDs. Thus, they offer color purity for lighting and displays, and enhanced gain for lasers. ${ }^{11-12}$

However, as prepared, NPLs are less photostable ${ }^{13}$ and exhibit a lower photoluminescence quantum yield (QY) than state-of-the-art QDs. The fluorescence of NPLs is also highly sensitive to their geometry ${ }^{14}$ and chemical environment. ${ }^{15}$ The best QDs exploit "core/shell" structures, in which a layer of another material is deposited to protect the exciton from the surface and reduce the interaction with surface trap states and the surrounding matrix. ${ }^{6}$ Moreover, protocols for growing a shell on the QD core have been continually improved and high-quality core/shell nanocrystals can 
now be produced for a large variety of shell types. ${ }^{4,16-18}$ Such protocols typically utilize extended reaction times, high reaction temperatures, and customized precursor and ligand combinations. This allows for the epitaxial growth of uniform crystalline shells with a smooth core/shell interface, good surface passivation, and near-unity QYs.

For NPLs, however, core/shell structures of such high quality with simultaneously stable, efficient, and narrow emission cannot yet be synthesized. The small thickness and anisotropic shape of NPLs lead to a lower thermodynamic stability compared to QDs. ${ }^{19}$ This renders them prone to dissolution at elevated temperatures, making high-temperature shell growth challenging. As an alternative approach, colloidal atomic layer deposition (c-ALD) at low temperature can be used to grow shells. ${ }^{20-21}$ This method allows for a wide range of possible core/shell combinations and avoids issues related to low thermal stability. Unfortunately, the resulting NPLs typically show relatively low QYs, and their synthesis requires many purification steps, essentially one after each atomic layer is added to the shell.

A few protocols do exist that employ a continuous growth of the shell at relatively low temperatures. While such core/shell NPLs achieved QYs up to $80 \%$, they suffer from limited reproducibility, and non-uniform and defective shell growth that broadens the emission. ${ }^{22-24}$ Presumably, these limitations are a result of the synthesis being performed at lower temperatures. Meeting the fundamental challenges associated with the stability of NPLs at high temperature may allow new and improved core/shell NPLs with higher QYs, uniformity, and stability to be obtained.

To move in this direction, we recently succeeded in growing CdS shells on CdSe NPLs at high temperatures. ${ }^{25}$ By improving the thermal stability of CdSe NPLs with the addition of cadmium oleate, we minimized the NPL dissolution at high temperature and obtained uniform and bright core/shell NPLs with narrow emission linewidths. In principle, this approach could also be applied to other shell types to create core/shell NPL structures with improved and tailored optical and electronic properties. However, a deeper understanding of the shell growth on such quasi-two-dimensional (quasi-2D) nanoplatelets is necessary to develop robust and efficient synthesis protocols. 
In the following, we address this need by investigating the high-temperature growth of a variety of shells on CdSe nanoplatelets. In particular, our synthesis protocol allows us to explore core/shell structures that have been well investigated in CdSe QDs. ${ }^{16-17,26-28}$ First, we discuss the effect of strain in $\mathrm{CdSe} / \mathrm{ZnS}$ core/shell NPLs and elucidate the limitations of $\mathrm{ZnS}$ in providing a uniform shell morphology for narrow emission linewidths. Our conclusions are verified by modifying the shell design towards a layered $\mathrm{CdS} / \mathrm{ZnS}$ shell. Second, to reduce the emission linewidth and improve the QY, we develop optimized $\mathrm{CdSe} / \mathrm{Cd}_{\mathrm{x}} \mathrm{Zn}_{1-\mathrm{x}} \mathrm{S}$ core/shell NPLs with a compositionally graded shell. Such core/shell NPLs exhibit high particle crystallinity and uniformity, narrow emission linewidths, and high QYs. We discuss the improved quality and characterize the structural and optical properties of these graded core/shell NPLs. Finally, we demonstrate the general flexibility of our coating protocol by synthesizing core/shell NPLs with a ZnSe shell. We conclude with a demonstration of the utility of our core/shell NPLs by examining their use in all-NPL ring lasers.

\section{RESULTS AND DISCUSSION}

CdSe/ZnS Core/Shell NPLs. As mentioned above, we previously developed a high-temperature protocol for the addition of uniform CdS shells to CdSe NPLs. ${ }^{25}$ However, the imperfect protection of the exciton from the outer surface can be a limiting factor for the photoluminescence efficiency of $\mathrm{CdSe} / \mathrm{CdS}$ core/shell NPLs. One solution is to exploit a larger-bandgap material for the shell. The exciton is then more strongly confined to the core, and any effects of an incomplete surface passivation can be reduced. ${ }^{29}$ This was recently implemented by synthesizing highly luminescent $\mathrm{CdSe} / \mathrm{ZnS}$ NPLs. ${ }^{24,30}$ However, the efficient photoluminescence in CdSe/ZnS NPLs came with broadening of the emission linewidth and an irregular shell growth.

Our first aim is to understand the growth process of the $\mathrm{ZnS}$ shell and avoid these problems. We synthesized CdSe/ZnS core/shell NPLs by a modification of our high-temperature protocol for CdS shells [see Figure S1 in the Supporting Information (SI)]. Similar to previous reports, we first observed a more irregular shell morphology along with an increased emission linewidth. CdSe and $\mathrm{ZnS}$ have a large lattice mismatch $(\sim 12 \%)$. As a result, increased strain with thicker $\mathrm{ZnS}$ shells can be released by 
forming defects and irregularities in the shell. ${ }^{31-33}$ However, under adjusted synthesis conditions (see Section S1 in the SI for details) we avoided this irregular shell growth and could reduce the emission linewidth (Figure 1a, Figures S2 and S3, and Table S1). Briefly, we improved the shell morphology by reducing the initial $\mathrm{Zn}$ precursor concentration and increasing the monomer solubility (by adding oleic acid). We hypothesized that such an approach would enhance the alloying of the core/shell interface and reduce strain. Indeed, this explanation is consistent with a detailed structural analysis of our improved NPLs by high-angle annular dark-field scanning transmission electron microscopy (HAADF STEM) and energy-dispersive X-ray spectroscopy (EDS) (Figure S4).

These experiments also indicated that the use of $\mathrm{Zn}(\text { oleate })_{2}\left[\mathrm{Zn}(\mathrm{OA})_{2}\right]$ instead of $\mathrm{Cd}(\text { oleate })_{2}$ $\left[\mathrm{Cd}(\mathrm{OA})_{2}\right]$ as the cation precursor affects the etching of the NPLs prior to shell growth. To examine this effect, we monitored the absorption spectrum and the shape of CdSe NPLs while heating to $300{ }^{\circ} \mathrm{C}$ with either $\mathrm{Cd}(\mathrm{OA})_{2}$ or $\mathrm{Zn}(\mathrm{OA})_{2}$. In the presence of $\mathrm{Zn}(\mathrm{OA})_{2}$, an increased broadening of the lowestenergy excitonic peak and an emergent absorption at wavelengths longer than the NPL excitonic features can be observed (Figure S5). An analysis of the corresponding HAADF STEM images shows no significant difference in the average lateral size at high temperatures (Figures S6 and S7). However, these images reveal a substantial change in the shape at high temperatures. In the presence of $\mathrm{Cd}(\mathrm{OA})_{2}$, the NPLs dissolve slowly and morph into uniform circular disks. In contrast, $\mathrm{Zn}(\mathrm{OA})_{2}$ enhances the facet-specific dissolution towards non-uniform shapes. Furthermore, a higher signal intensity was recorded in HAADF STEM at the edges of the NPLs that were treated in $\mathrm{Zn}(\mathrm{OA})_{2}$ at high temperature, indicating an increased thickness. This irregular material transfer to the edges can explain the increased absorption at longer wavelengths and the broader excitonic features measured for these aliquots. Such an anisotropic material transfer induced by $\mathrm{Zn}(\mathrm{OA})_{2}$ has also been observed previously on other nanocrystals. ${ }^{34}$ We conclude that a combination of high strains in CdSe/ZnS NPLs and the anisotropic etching induces the emission broadening and the irregular shell morphology. By adjusting the shell growth conditions accordingly, these effects could be reduced in CdSe/ZnS NPLs (Figure 1a).

CdSe/CdS/ZnS Core/Shell/Shell NPLs. To test this understanding further, we coated CdSe NPLs with CdS before the growth of $\mathrm{ZnS}$. In this heterostructure, CdS reduces strain by bridging the 
lattice mismatch between CdSe and ZnS. Indeed, for QDs such a shell structure resulted in better optical properties, while maintaining narrow emission linewidths of $\sim 77 \mathrm{meV}(\sim 24 \mathrm{~nm}$ at $\sim 620 \mathrm{~nm}) .{ }^{17}$ In addition, for NPLs the use of $\mathrm{Cd}(\mathrm{OA})_{2}$ as the precursor for the $\mathrm{CdS}$ shell growth avoids the anisotropic etching of the CdSe core. To obtain CdSe/CdS/ZnS NPLs, we coated CdSe NPLs first with CdS by the slow injection of 1 -octanethiol at $300^{\circ} \mathrm{C}$ into a mixture of CdSe NPLs and Cd(OA)2. This step was then followed by a second slow injection of 1 -octanethiol and $\mathrm{Zn}(\mathrm{OA})_{2}$ at $280{ }^{\circ} \mathrm{C}$. Figure $1 \mathrm{~b}$ shows a HAADF STEM image of such CdSe/CdS/ZnS NPLs. They have a uniform morphology with a shell thickness of approximately $2-3 \mathrm{~nm}$. Interestingly, we noticed an improved colloidal stability of the final core/shell/shell NPLs after the addition of ZnS. This suggests that the ligands are bound more strongly on the NPLs capped with $\mathrm{ZnS}$ than $\mathrm{CdS} .{ }^{35}$ After the $\mathrm{ZnS}$ overcoating, we also measured an increase in the QY from $60-70 \%$ in CdSe/CdS NPLs up to $\sim 80 \%$ in CdSe/CdS/ZnS NPLs.

CdSe/Cd $Z_{\mathbf{x}} \mathbf{n}_{1-\mathbf{x}} \mathbf{S}$ Core/Shell NPLs. The above data demonstrate the advantage of combining $\mathrm{CdS}$ and $\mathrm{ZnS}$ in the shell and indicate the dual benefit of strain reduction and improved surface passivation. This suggests that the ideal shell would contain a gradual increase in concentration of $\mathrm{ZnS}$ towards the surface. We therefore designed a synthesis protocol for a compositionally graded alloyed shell with a Cd-rich inner part and a Zn-rich outer part. It has been shown that cadmium chalcogenides grow on CdSe at a higher rate than the corresponding zinc chalcogenides. ${ }^{36-37}$ This difference in the reaction rates enabled us to obtain the desired composition gradient by combining CdSe NPLs with $\mathrm{Cd}(\mathrm{OA})_{2}$ and $\mathrm{Zn}(\mathrm{OA})_{2}$ in different ratios. The shell synthesis was performed at $300{ }^{\circ} \mathrm{C}$ by slowly injecting 1-octanethiol together with oleic acid. Figure $1 \mathrm{c}$ shows such $\mathrm{CdSe} / \mathrm{Cd}_{\mathrm{x}} \mathrm{Zn}_{1-\mathrm{x}} \mathrm{S}$ core/shell NPLs using a $\left[\mathrm{Cd}(\mathrm{OA})_{2}\right]:\left[\mathrm{Zn}(\mathrm{OA})_{2}\right]$ precursor ratio of 1:2. This approach resulted in crystalline and uniform shells with a thickness of 1.5-2 nm. Compared to the original synthesis of CdSe/CdS NPLs, nearly no formation of side products was observed. We attribute this reduced homogeneous nucleation to a higher monomer solubility due to the injection of oleic acid and the lower reactivity of the $\mathrm{Zn}$ precursor.$^{37-38}$ We discuss the optimization and characterization of $\mathrm{CdSe} / \mathrm{Cd}_{\mathrm{x}} \mathrm{Zn}_{1-\mathrm{x}} \mathrm{S}$ core/shell NPLs further in a later section. 
CdSe/ZnSe Core/Shell NPLs. In principle, such a graded composition can be synthesized also with other shell materials. However, to the best of our knowledge, protocols for coating NPLs with a uniform shell have so far been limited to sulfide shells. By synthesizing novel shell types, the available core/shell NPL systems can be extended, which allows additional control over the nanoplatelet properties. We therefore examined $\mathrm{ZnSe}$ as an additional shell material. Because of the smaller difference in the lattice constants between CdSe and $\mathrm{ZnSe}$, we expected a more uniform shell growth than for CdSe/ZnS NPLs.

For the Se precursor, we used a solution of Se dissolved in 1-octadecene (Se-ODE). To obtain $\mathrm{CdSe} / \mathrm{ZnSe}$ core/shell NPLs, we then slowly injected Se-ODE together with oleic acid at different temperatures between $260{ }^{\circ} \mathrm{C}$ and $300{ }^{\circ} \mathrm{C}$ into a mixture of CdSe NPLs and $\mathrm{Zn}(\mathrm{OA})_{2}$. Because SeODE exhibited higher reactivity than 1-octanethiol (the sulfur precursor used above), the control of the shell growth was more challenging, and we observed substantial homogeneous nucleation of $\mathrm{ZnSe}$ nanocrystals. Figure $1 \mathrm{~d}$ shows the NPLs resulting from the synthesis at $300{ }^{\circ} \mathrm{C}$. We measured a shell thickness of 2-3 nm in these CdSe/ZnSe NPLs, which is slightly larger than for NPLs with ZnS shells. This faster growth can be attributed to a higher reactivity of Se-ODE compared to 1-octanethiol, leading to a faster and more complete precursor conversion.

When the composition was analyzed by EDS, the Cd was found far into the shell despite the absence of $\mathrm{Cd}$ as a precursor during the shell growth (Figure S8). Similar to the case of ZnS shells, this may be explained by a partial dissolution of the CdSe NPL cores and the subsequent incorporation of this material into the shell (see discussion in Section S1 in the SI). Furthermore, since only the cation is altered between core and shell, the high reaction temperature is expected to accelerate the cationic diffusion near the core/shell interface. ${ }^{39}$

X-Ray-Diffraction (XRD) Analysis. XRD results can complement the characterization of the crystal structure performed by electron microscopy. All of our core/shell NPLs show only zinc blende (zb) diffraction peaks, indicating that the shell adopts the crystal structure of the core (Figure 2). However, differences in the peak width and shape can be observed. While the CdSe/CdS/ZnS NPLs show the narrowest diffraction signals, the peaks broaden substantially in the CdSe/ZnS NPLs. 
Partially, this is caused by the difference in lateral size and thickness of the NPLs, which affects the Scherrer line broadening. However, strain in the core/shell NPLs also contributes to the peak broadening. CdSe has a larger lattice constant than the material in the shell. Thus, a higher content of $\mathrm{Zn}$ and/or S in the shell strains the NPLs more. This results in a spread of the diffraction angles and broadens the pattern.

In addition, the core/shell structure also affects the symmetry of the diffraction peaks. The larger lattice constant in the core contributes to the diffraction signal at smaller angles. However, the smaller volume of the core compared to the shell reduces its contribution to the pattern and explains the observed asymmetry of the diffraction peaks [most obvious for the (220)-reflections of $\mathrm{CdSe} / \mathrm{Cd}_{\mathrm{x}} \mathrm{Zn}_{1-\mathrm{x}} \mathrm{S}$ and $\mathrm{CdSe} / \mathrm{ZnS}$ NPLs near $\left.43^{\circ}\right]$. Furthermore, biaxial strain in the core/shell NPLs owing to the quasi-2D geometry of the core may increase the peak asymmetry. ${ }^{40}$

Optical Characterization of Core/Shell NPLs. The influence of the shell composition on the emission was characterized by absorption and emission spectroscopy (Figure 3). We achieved narrow emission linewidths below $60 \mathrm{meV}$ [full-width-at-half-maximum (FWHM)] for our core/shell NPLs. The smallest values were measured for graded $\mathrm{CdSe} / \mathrm{Cd}_{\mathrm{x}} \mathrm{Zn}_{1-\mathrm{x}} \mathrm{S}$ core/shell NPLs (56 meV, Table S1). For 4-monolayer-thick-CdSe/Cd $\mathrm{Cd}_{\mathrm{x}} \mathrm{Zn}_{1-\mathrm{x}} \mathrm{S}$ NPLs emitting at $655 \mathrm{~nm}$, this linewidth corresponds to a $19.5 \mathrm{~nm}$ FWHM (Table S1). Of our different optimized NPLs, only CdSe/ZnS core/shell NPLs showed a significantly broader emission peak with a FWHM of $\sim 76 \mathrm{meV}(\sim 23.7 \mathrm{~nm}$ at $620 \mathrm{~nm}$, Table $\mathrm{S} 1)$.

For $\mathrm{CdSe} / \mathrm{CdS} / \mathrm{ZnS}$ NPLs we measured emission wavelengths centered at 660-670 nm. Slightly shorter values were obtained for $\mathrm{CdSe} / \mathrm{Cd}_{\mathrm{x}} \mathrm{Zn}_{1-\mathrm{x}} \mathrm{S}$ NPLs where we measured $655 \mathrm{~nm}$ for a precursor ratio of $\mathrm{Cd}(\mathrm{OA})_{2}: \mathrm{Zn}(\mathrm{OA})_{2}=1: 2$. This can be attributed to a small difference in the shell thickness and a different $\mathrm{Cd}$ content in the shell. Reducing $\mathrm{Cd}$ (in favor of $\mathrm{Zn}$ ) increases the exciton confinement, which leads to emission at shorter wavelengths. Hence, the shortest values were measured for $\mathrm{CdSe} / \mathrm{ZnS}$ NPLs at $\sim 620 \mathrm{~nm}$ for smooth $\mathrm{ZnS}$ shells. We also observed an increase in the photoluminescence lifetime with higher Cd content in the shell (Figure S9 and Table S1). 
Interestingly, CdSe/ZnS NPLs with shells synthesized at room temperature by c-ALD emit at wavelengths shorter than $600 \mathrm{~nm}$, even with thicker shells. ${ }^{24}$ This implies a structural difference in the shell or at the interface. The incorporation of material from the core into the shell for our $\mathrm{CdSe} / \mathrm{ZnS}$ NPLs (as observed by EDS, Figure S4) can explain the more pronounced red-shift that we observe. For example, more $\mathrm{Cd}$ in the shell increases the exciton delocalization. Furthermore, compressive strains at sharper interfaces may cause a blue-shift of the emission. Consequently, for our CdSe/ZnS NPLs that have an irregular shell morphology, emission occurred near $610 \mathrm{~nm}$ (Figure S3).

Our CdSe/ZnSe NPLs showed an emission highly sensitive to the reaction temperature. Emission wavelengths were measured from $\sim 630$ to $685 \mathrm{~nm}$ with the longest values obtained for reactions performed at the highest temperature of $300^{\circ} \mathrm{C}$. Notably, such a large emission red-shift of $170 \mathrm{~nm}$ $(0.6 \mathrm{eV})$ was achieved while retaining narrow emission having a FWHM of only $22 \mathrm{~nm}(59 \mathrm{meV}$, Table S1). We explain this emission red-shift with the high reaction temperature that accelerates both the partial dissolution of the CdSe NPL core followed by its incorporation into the shell and the cation diffusion near the core/shell interface. This results in a reduction of the potential well in the inner part of the shell and enhances the delocalization of the exciton wave function.

Optimization of $\mathrm{CdSe} / \mathrm{Cd}_{\mathrm{x}} \mathrm{Zn}_{1-\mathrm{x}} \mathrm{S}$ Core/Shell NPLs. To rationalize the properties of the $\mathrm{Cd}_{\mathrm{x}} \mathrm{Zn}_{1-\mathrm{x}} \mathrm{S}$ shells, we examined the synthesis of these materials further. As discussed above, the spectral position of the emission can be controlled by tuning the ratio between $\mathrm{Cd}$ and $\mathrm{Zn}$. Thus, we prepared core/shell NPLs using different cation precursor ratios and precursor quantities. The molar fraction of $\mathrm{Cd}(\mathrm{OA})_{2}$ (relative to the total amount of metal oleate) was varied between 0 and $100 \%$. Reactions were then performed with a total precursor amount (Cd plus $\mathrm{Zn}$ ) of $0.05,0.1$, and $0.2 \mathrm{mmol}$ metal oleates (the molar ratio between metal oleates and 1-octanethiol was fixed at 1:1.2). The results show that a larger amount of total precursor led to a stronger emission red-shift (Figure 4a). This is expected for the formation of a thicker shell and, therefore, weaker exciton localization. The emission wavelength is also clearly dependent on the precursor ratio. Increasing the fraction of $\mathrm{Cd}(\mathrm{OA})_{2}$ up to around $25-30 \%$ shifts the emission steadily towards longer wavelengths. However, in all concentration series we observed no further red-shift beyond a $\mathrm{Cd}(\mathrm{OA})_{2}$ fraction of $30 \%$. This 
indicates that a relatively large fraction of $\mathrm{Zn}(\mathrm{OA})_{2}$ is required to achieve changes in the shell composition and, hence, the emission behavior. In fact, for a $\mathrm{Cd}: \mathrm{Zn}$ precursor ratio of 1:2 we measured a concentration ratio for $\mathrm{Cd}: \mathrm{Zn}$ of $3.88: 1$ in the final core/shell NPLs by Rutherford back scattering (RBS) (Figure S10a). Subtracting the material in the CdSe core (Cd:Se ratio of 1.30:1, Figure S10b) results in a Cd:Zn ratio of $\sim 2.8: 1$ in the shell.

The data show that the precursor ratio and the final shell thickness also affect the emission linewidth (Figure $4 \mathrm{~b}, \mathrm{c})$. The addition of more $\mathrm{Cd}(\mathrm{OA})_{2}$, either by adding more precursor or increasing the Cd:Zn molar ratio, results in thicker shells and narrower emission peaks, reaching linewidths below $60 \mathrm{meV}$ (FWHM). This value corresponds to those measured for CdSe/CdS core/shell NPLs obtained via the c-ALD approach (shaded area in Figure 4b) in which the shell is expected to grow with atomic precision. ${ }^{20-21}$ Thicker shells also minimize variations in the emission linewidth, as indicated in Figure $4 \mathrm{c}$ by the encircled clusters of black and red data points for reactions with $\left[\mathrm{Cd}(\mathrm{OA})_{2}\right] \geq 25 \%$. Our linewidth narrowing can be explained by an improved shell uniformity achieved for thicker shells (as observed in pure CdS shells ${ }^{25}$ ) and reduced strains and defects. ${ }^{41}$ Strains due to an increased lattice mismatch explain also the broader emission with linewidths up to $100 \mathrm{meV}$ for the syntheses of $\mathrm{ZnS}$ shells at a higher precursor concentration. (As discussed above, this leads to the formation of irregular shell morphologies.) No clear trend could be observed for the reaction series with $0.05 \mathrm{mmol}$ metal oleate. In such thin shells, strain plays a smaller role and we can obtain even narrower emission linewidths with $\mathrm{ZnS}$ than with $\mathrm{CdS}$ shells. Instead, the stronger exciton confinement with thinner shells renders the linewidth more sensitive to synthesis conditions and its spread becomes generally larger.

Structural and Optical Characterization of $\operatorname{CdSe/Cd} \mathbf{Z n}_{1-x} S$ Core/Shell NPLs. We expect that the simultaneous addition of $\mathrm{Cd}(\mathrm{OA})_{2}$ and $\mathrm{Zn}(\mathrm{OA})_{2}$ at the beginning of the shell synthesis allows the formation of epitaxial, compositionally graded $\mathrm{Cd}_{\mathrm{x}} \mathrm{Zn}_{1-\mathrm{x}} \mathrm{S}$ shells. To verify this, we performed a detailed structural analysis of these core/shell NPLs. Figure 5a shows a cross-sectional view of a $\mathrm{CdSe} / \mathrm{Cd}_{\mathrm{x}} \mathrm{Zn}_{1-\mathrm{x}} \mathrm{S}$ NPL along the [100]-direction at atomic resolution acquired by STEM. The image shows that the shell adopts the lattice of the core, forming an epitaxial interface. The heavier and brighter core can be distinguished clearly from the shell due to the difference in composition. In 
addition, the elemental profile of such core/shell NPLs can be obtained by EDS for top and side views (Figure 5b). Figure 5c shows the elemental concentration profile across a stack of 4 NPLs, observed from the side. The profile is extracted in the direction of the white arrow in Figure $5 \mathrm{~b}$. It reveals a spatially dependent $\mathrm{Zn}$ concentration, increasing with the distance from the CdSe core. Hence, these data are consistent with the formation of an epitaxial and compositionally graded shell.

Combining this structural information with the resulting optical properties allows one to draw conclusions for the design of optimized core/shell NPLs. In particular, besides the effect on the emission linewidth we further evaluated the impact of the shell design on the QY, a key quantity for many applications. Figure 6a shows the QY as a function of excitation wavelength for $\mathrm{CdSe} / \mathrm{Cd}_{\mathrm{x}} \mathrm{Zn}_{1-\mathrm{x}} \mathrm{S}$ NPLs for which the shell was grown with a precursor ratio $\left[\mathrm{Cd}(\mathrm{OA})_{2}\right]:\left[\mathrm{Zn}(\mathrm{OA})_{2}\right]$ of 1:2. These NPLs exhibited narrow emission while containing a relative high amount of $\mathrm{Zn}$ in the outer part of the shell. A very high QY of $90 \%$ that is nearly independent of the excitation wavelength was achieved without additional surface treatment after the synthesis.

The data suggest that the high QY values observed for these graded shells result from several factors. First, the presence of a ZnS-rich outer layer improves the passivation and protection from the environment compared to a pure CdS shell. This can explain why we measured lower QY values (6070\%) for our CdSe/CdS NPLs. Similarly, incomplete surface passivation may also be limiting the QY of our CdSe/ZnSe NPLs, for which we measured values near 50\% (Table S1). We expect that an increase of the QY for CdSe/ZnSe NPLs may be possible with an improved surface passivation, possibly by adding ZnS or a better ligand coverage. The second factor that can lead to high QY in our optimized $\mathrm{Cd}_{\mathrm{x}} \mathrm{Zn}_{1-\mathrm{x}} \mathrm{S}$ shells is their high Cd content in the part of the shell close to the core/shell interface. Compared with a pure, unalloyed $\mathrm{ZnS}$ shell, this reduces the lattice mismatch and minimizes defect formation, allowing a narrow emission peak with high QY to be obtained. (We assume that the reported high QY values in CdSe/ZnS NPLs ${ }^{30}$ also result from partial alloying at the interface.) The third factor is the high reaction temperature and slow shell growth, which can reduce lattice strain and defects by atomic diffusion. ${ }^{42-43}$ A similar behavior was observed in $\mathrm{CdSe} / \mathrm{CdS}$ nanorods, where a 
slow over-coating of the initially slightly defective shell at high temperatures allowed for an increase of the QY to near unity. ${ }^{44}$

To confirm the emission stability of our $\mathrm{CdSe} / \mathrm{Cd}_{x} \mathrm{Zn}_{1-\mathrm{x}} \mathrm{S}$ NPLs we recorded the photoluminescence of NPLs excited at different excitation wavelengths (400 to $640 \mathrm{~nm}$, Figure 6b) and fluences (10 to $1000 \mu \mathrm{J} / \mathrm{cm}^{2}$, Figure $6 \mathrm{c}$ ). The core/shell NPLs show a stable emission peak that is nearly independent of the excitation wavelength and power, even for the highest fluences.

We also note that, because the direct synthesis of thicker 6-monolayer-thick CdSe NPLs was recently reported, ${ }^{45-46}$ we tested the versatility of our shell protocol and applied it to these NPLs. The resulting $\mathrm{CdSe} / \mathrm{Cd}_{\mathrm{x}} \mathrm{Zn}_{1-\mathrm{x}} \mathrm{S}$ core/shell NPLs showed a narrow emission peak with a FWHM of $57 \mathrm{meV}$ $(22 \mathrm{~nm})$ and a strong red-shift to $692 \mathrm{~nm}$. The corresponding absorption and emission spectra, HAADF STEM images, and EDS data are shown in Figures S11 and S12. These core/shell NPLs exhibit very high QYs between 90 and 95\% and exceptional emission stability (Figure 6d-f). Hence, such compositionally graded $\mathrm{CdSe} / \mathrm{Cd}_{\mathrm{x}} \mathrm{Zn}_{1-\mathrm{x}} \mathrm{S}$ core/shell NPLs reveal the benefit of strain reduction and surface passivation for improved optical performance.

Nanoplatelet Ring Lasers. The properties discussed above illustrate the potential of the core/shell NPLs for applications that rely on narrow emission with high QY. However, the suitability for more complex optical devices remains to be examined. Therefore, as a demonstration, we evaluated the performance of our different core/shell NPL structures for lasing applications.

In semiconductor nanocrystals, non-radiative Auger recombination can be a critical loss mechanism competing with population inversion and lasing. ${ }^{47}$ However, it has been shown that Auger recombination can be significantly slowed by utilizing core/shell nanocrystals with compositionally graded core/shell interfaces. ${ }^{48-49}$ In addition, the relaxation from 3D to $1 \mathrm{D}$ exciton confinement in NPLs may further reduce the Auger recombination rate by enforcing a stricter momentum conservation. In this context, NPLs have shown great potential as an active gain material for lasers. Low-threshold lasing under pulsed and continuous wave photoexcitation have recently been reported for bare CdSe NPLs and NPLs coated with a shell by c-ALD. ${ }^{50-53}$ Core/shell NPLs with a 
compositionally graded shell may provide further improvements in performance, stability, and variability compared to bare NPLs.

For our evaluation, we chose ring resonators as a platform because of their simple architecture. ${ }^{54}$ Namely, we used patterned films of core/shell NPLs that contained circular waveguides (Figure 7a,b). In this design, the NPLs create both the ring resonator and provide the gain material. We fabricated such structures by template-stripping films of core/shell NPLs from pre-patterned silicon templates. ${ }^{55}$ As we showed previously for QDs, ${ }^{55}$ this method allows for rapid production of structures with high quality and variable geometry. To achieve population inversion and lasing, the rings were then illuminated by ultrafast laser pulses at a wavelength of $405 \mathrm{~nm}$. Figure $7 \mathrm{c}$ shows the resulting powerdependent emission spectra of a 4-monolayer-CdSe/Cdx $\mathrm{Zn}_{1-\mathrm{x}} \mathrm{S}$ NPL ring laser. The transition from spontaneous emission to multimode lasing can be observed as an abrupt increase of the emission peak intensity and the appearance of a series of sharp lasing peaks (cavity modes) above a threshold of $13 \mu \mathrm{J} / \mathrm{cm}^{2}$ (Figure $7 \mathrm{~d}, \mathrm{e}$ ). This threshold is lower than values measured for core/shell QDs using the same ring structures. ${ }^{54}$ Our different core/shell NPL ring lasers all showed lasing modes at wavelengths between $\sim 630$ (CdSe/ZnS NPLs) and $\sim 720 \mathrm{~nm}$ (6-monolayer-CdSe/CdxZn1-xS NPLs), and low threshold fluences below $50 \mu \mathrm{J} / \mathrm{cm}^{2}$ (Figure S13). Our analysis supports the potential of such core/shell NPLs for lasing applications. Despite the simple structure, we achieved low thresholds and stable lasing performance up to very high excitation powers. We were able to sustain lasing for excitation powers ranging over $\sim 2$ orders of magnitude. Future work on the influence of the shell structure on the Auger recombination rate may help optimize these core/shell NPLs further for lasing applications.

\section{CONCLUSIONS}

We have developed a high-temperature, slow-injection shell-growth protocol that can produce core/shell nanoplatelets with various shell designs. The structural and optical characterization of the resulting materials revealed the crucial role of a compositional gradient in nanoplatelet shells. Such a design helps avoid the formation of strain-induced defects in these anisotropic nanocrystals and 
enables high emission efficiencies and stability while retaining narrow emission linewidths. As a consequence, we achieved spectrally narrow and tunable emission, and reached QYs above 90\%, overcoming previous limitations in NPL-based core/shell structures. In addition, such control over the shell composition helped us achieve low lasing thresholds in simple architectures with stable output over large power ranges. These core/shell NPLs can potentially impact applications relying on narrow, bright, efficient, and stable emission. Furthermore, owing to their unique geometry, such core/shell structures may be ideal for fundamental studies of diffusion processes, strain effects, and bandstructure engineering in nanocrystals.

\section{EXPERIMENTAL SECTION}

Materials and Reagents. 1-octadecene (ODE, 90\%, \#O806), 1-octanethiol ( $\geq 98.5 \%$, \#471836), 2-propanol ( $\geq 99.9 \%, \# 34965)$, acetonitrile ( $\geq 99.9 \%, \# 34998)$, cadmium acetate hydrate $\left[\mathrm{Cd}(\text { acetate })_{2}\right.$ hydrate, $\geq 99.99 \%$, \#229490], cadmium acetate dihydrate [Cd(acetate)2 dihydrate, 98\%, \#289159], methyl acetate ( $\geq 98 \%$, \#W267600), cadmium chloride (technical grade, \#655198), chloroform (99.8\%,\#132950), $n$-hexane ( $\geq 97.0 \%, \# 34859)$, methylcyclohexane (MCH, 99\%, \#M37889), myristic acid ( $\geq 98 \%$, \#70082), octane (98\%, \#412236), oleic acid (OA, 90\%, \#364525), selenium ( $\geq 99.5 \%$, $\# 209651)$, trifluoroacetic acid ( $\geq 99.0 \%$, \#302031), trifluoroacetic anhydride ( $\geq 99 \%$, \#106232), zinc acetate $(99.99 \%, \# 383317)$, and zinc oxide $(\geq 99.0 \%$, \#96479) were purchased from Sigma-Aldrich. Octadecyltrichlorosilane (ODTS, 96\%) was purchased from Merck KGaA. Cadmium oxide (99.999\%, \#48-0800) was obtained from Strem Chemicals. Oleylamine (OAm, 80-90\%, \#129541000) and triethylamine (99\%, \#157910010) were acquired from Acros Organics. Methanol was purchased from Thommen-Furler AG and absolute ethanol from Alcosuisse AG. Four-inch diameter, single-sidepolished, single-crystalline $\mathrm{Si}(100)$ wafers with $<0.4 \mathrm{~nm}$ root-mean-square (RMS) roughness and thicknesses of either 500 or $1000 \mu \mathrm{m}$ were purchased from Silicon Valley Microelectronics or Silicon Quest and diced into $2 \times 2 \mathrm{~cm}^{2}$ square pieces. All chemicals were used without further purification, except where noted. 
Preparation of Cadmium Myristate [Cd(myristate $\left.)_{2}\right]$, Cadmium Oleate $\left[\mathrm{Cd}(\text { oleate) })_{2}\right]$, and Zinc Oleate $\left[\mathbf{Z n}(\text { oleate })_{2}\right]$. Cadmium carboxylates and zinc oleate were synthesized following a modified protocol by Hendricks et al. ${ }^{56}$ Briefly, $5.75 \mathrm{~g} \mathrm{CdO}(3.65 \mathrm{~g} \mathrm{ZnO})$ and $20 \mathrm{~mL}$ acetonitrile were mixed in a $100-\mathrm{mL}$ round-bottom flask. The mixture was then stirred and cooled in an ice bath. Subsequently, $6.2 \mathrm{~mL}$ of trifluoroacetic anhydride, and $0.7 \mathrm{~mL}$ trifluoroacetic acid were added. After $10 \mathrm{~min}$, the ice bath was removed and the flask heated at $50{ }^{\circ} \mathrm{C}$ until the solution turned clear. In a 500-mL Erlenmeyer flask, $180 \mathrm{~mL}$ 2-propanol, $14.0 \mathrm{~mL}$ of triethylamine, and $20.6 \mathrm{~g}$ myristic acid (or $28.6 \mathrm{~mL}$ oleic acid) were mixed and stirred. The warm cadmium trifluoroacetate (or zinc trifluoroacetate) solution was then slowly added to the myristic acid (or oleic acid) solution while stirring. The resulting white precipitate was vacuum-filtered using a fritted glass funnel and rinsed thoroughly with methanol. The final product was dried in a vacuum oven over night and stored under ambient conditions.

Preparation of Zinc Oleate Stock Solution. $367 \mathrm{mg} \mathrm{Zn(acetate)2,} 7 \mathrm{~mL}$ ODE, and $1.2 \mathrm{~mL}$ OA were mixed in a glass jar in a $\mathrm{N}_{2}$-filled glovebox. The mixture was then stirred at $220{ }^{\circ} \mathrm{C}$ for $2 \mathrm{~h}$. Afterwards, it was allowed to cool to room temperature (RT). While cooling, 1.3 mL OAm was added at around $100{ }^{\circ} \mathrm{C}$ to prevent solidification. The final product was stored in a $\mathrm{N}_{2}$-filled glovebox.

Preparation of Se-ODE Stock Solution. $79 \mathrm{mg}$ Se powder (1 mmol) (stored in a $\mathrm{N}_{2}$-filled glove box) and $10 \mathrm{~mL}$ ODE was added to a $50-\mathrm{mL}$ round-bottom flask and degassed at RT under vacuum for $30 \mathrm{~min}$. The mixture was then heated to $240{ }^{\circ} \mathrm{C}$ under $\mathrm{N}_{2}$ and kept for $30 \mathrm{~min}$. Afterwards, it was allowed to cool to RT and transferred to a $\mathrm{N}_{2}$-filled glovebox.

Synthesis of 4-Monolayer-Thick CdSe NPLs. For the CdSe NPL synthesis, $680 \mathrm{mg}$ Cd(myristate)2, $48 \mathrm{mg}$ Se powder (stored in a $\mathrm{N}_{2}$-filled glove box), and $60 \mathrm{~mL}$ ODE were added to a 250-mL round-bottom flask and degassed at RT under vacuum for $30 \mathrm{~min}$. Then, the mixture was heated to $240{ }^{\circ} \mathrm{C}$ under $\mathrm{N}_{2}$. At $200{ }^{\circ} \mathrm{C}, 256 \mathrm{mg} \mathrm{Cd}$ (acetate) 2 dihydrate was added. The mixture was kept at $240{ }^{\circ} \mathrm{C}$ for $8-9 \mathrm{~min}$. Afterwards, the reaction flask was cooled using an air gun to $160{ }^{\circ} \mathrm{C}$. During this cooling step, $3 \mathrm{~mL}$ OA was added when the temperature reached $185^{\circ} \mathrm{C}$. Once at $130{ }^{\circ} \mathrm{C}$, the flask was placed in a water bath and cooled to RT. Then, $10 \mathrm{~mL}$ hexane was added and the mixture 
was centrifuged at $8000 \mathrm{rpm}(8586 \mathrm{~g}$ ) for $10 \mathrm{~min}$. The precipitate was re-dispersed in $20 \mathrm{~mL}$ hexane and kept undisturbed for $1 \mathrm{~h}$. Afterwards, it was centrifuged at $7000 \mathrm{rpm}(6574 \mathrm{~g})$ for $7 \mathrm{~min}$. Unwanted 3-monolayer-thick NPLs were removed as the precipitate. The 4-monolayer-thick NPLs in the supernatant were then precipitated from the hexane dispersion by adding methyl acetate and centrifuging at $6500 \mathrm{rpm}(5668 \mathrm{~g})$ for $3 \mathrm{~min}$. The precipitate was re-dispersed in $6 \mathrm{~mL}$ hexane and stored in the dark under ambient conditions until needed.

Synthesis of 6-Monolayer-Thick CdSe NPLs. The synthesis of 6-monolayer CdSe NPLs was adapted from Cho et al. ${ }^{46}$ Briefly, $170 \mathrm{mg} \mathrm{Cd}$ (myristate)2 and $14 \mathrm{~mL}$ ODE were mixed in a $100-\mathrm{mL}$ round-bottom flask and degassed at $85{ }^{\circ} \mathrm{C}$ under vacuum for $30 \mathrm{~min}$. Then, the mixture was heated up to $250{ }^{\circ} \mathrm{C}$ under $\mathrm{N}_{2}$. At $250^{\circ} \mathrm{C}, 12 \mathrm{mg}$ Se powder (stored in a $\mathrm{N}_{2}$-filled glove box) dispersed in $1 \mathrm{~mL}$ ODE was quickly injected (the dispersion was sonicated for $20 \mathrm{~min}$ ). $20 \mathrm{~s}$ later, $60 \mathrm{mg} \mathrm{Cd(acetate})_{2}$ hydrate was added. After $1 \mathrm{~min}, 0.15 \mathrm{~mL}$ aqueous $\mathrm{CdCl}_{2}$ solution $(0.5 \mathrm{M})$ was added dropwise over a period of 90 seconds. The mixture was then kept for an additional 6 min at $250{ }^{\circ} \mathrm{C}$. Afterwards, the reaction flask was cooled to RT using an air gun. During this cooling step, $2 \mathrm{~mL}$ OA mixed with $15 \mathrm{~mL} \mathrm{MCH}$ was added slowly when the temperature reached $180^{\circ} \mathrm{C}$. For the purification, the mixture was first centrifuged for $10 \mathrm{~min}$ at $8500 \mathrm{rpm}(9693 \mathrm{~g})$. The precipitate was re-dispersed in $1.5 \mathrm{~mL}$ $\mathrm{MCH}$ and centrifuged at $6500 \mathrm{rpm}(5668 \mathrm{~g})$ for $2 \mathrm{~min}$. Salts and aggregated NPLs were removed as the precipitate. $3 \mathrm{~mL}$ hexane and $6 \mathrm{~mL}$ methyl acetate were then added to the 6-monolayer-thick NPLs in the supernatant and the mixture was centrifuged at $8000 \mathrm{rpm}(8586 \mathrm{~g})$ for $7 \mathrm{~min}$. The precipitate was re-dispersed in $1 \mathrm{~mL}$ hexane and stored in the dark under ambient conditions until needed. A stable reaction temperature is necessary for a high yield of 6-monolayer-thick NPLs. Otherwise, unwanted 5-monolayer-thick NPLs that form during the synthesis can be removed selectively as precipitate by centrifuging the final dispersion at $8000 \mathrm{rpm}(8586 \mathrm{~g})$ for $30 \mathrm{~min}$.

Synthesis of $\mathbf{C d S e} / \mathbf{C d}_{\mathbf{x}} \mathbf{Z n}_{1-\mathrm{x}} \mathrm{S}$ Core/Shell NPLs. In a typical synthesis, 4-monolayer CdSe NPLs [an amount equivalent to $1 \mathrm{~mL}$ of NPL dispersion with an optical density (OD) of 120 at the lowest energy excitonic peak, assuming a 1-cm optical path length], $10 \mathrm{~mL}$ ODE, $400 \mu \mathrm{L}$ OA, $90 \mathrm{mg}$ $\mathrm{Cd}($ oleate) 2 and $167.5 \mathrm{mg} \mathrm{Zn(oleate)} 2$ (total of $0.4 \mathrm{mmol}$ metal oleate, molar ratio of $1: 2$ ) were added 
to a $100-\mathrm{mL}$ round-bottom flask. The mixture was degassed under vacuum for $35 \mathrm{~min}$ at $\mathrm{RT}$ and $15 \mathrm{~min}$ at $80^{\circ} \mathrm{C}$. Afterwards, $2 \mathrm{~mL}$ OAm (stored in a $\mathrm{N}_{2}$-filled glovebox) was added and the temperature was raised to $300{ }^{\circ} \mathrm{C}$ under $\mathrm{N}_{2}$ at a rate of $\sim 15^{\circ} \mathrm{C} / \mathrm{min}$. Starting at $165{ }^{\circ} \mathrm{C}$, a solution of $83 \mu \mathrm{L}$ 1-octanethiol $(0.48 \mathrm{mmol})$ dissolved in $7 \mathrm{~mL}$ ODE and $2 \mathrm{~mL} \mathrm{OA}$ was injected at a rate of $4.5 \mathrm{~mL} / \mathrm{h}$. After complete injection, the solution was kept at $300{ }^{\circ} \mathrm{C}$ for $40 \mathrm{~min}$. Subsequently, the product was cooled down, and, at $40^{\circ} \mathrm{C}, \sim 5 \mathrm{~mL}$ hexane was added.

The core/shell NPLs were purified by selective precipitation. In a first step, the mixture was centrifuged at $6500 \mathrm{rpm}(5668 \mathrm{~g}$ ) for $6 \mathrm{~min}$. The core/shell NPLs in the precipitate were re-dispersed in $5 \mathrm{~mL}$ hexane. [If a significant amount of core/shell NPLs remained in the supernatant after this centrifugation step, they can be precipitated by adding methyl acetate and centrifuging at $6500 \mathrm{rpm}$ (5668 g).] Then, $\sim 5 \mathrm{~mL}$ methyl acetate was added to the dispersion of core/shell NPLs in hexane until the mixture turned turbid and it was centrifuged at $6500 \mathrm{rpm}(5668 \mathrm{~g})$ for $10 \mathrm{~min}$. This process (i.e. dispersion of the NPLs in hexane followed by precipitation with methyl acetate) was repeated. The core/shell NPLs were then re-dispersed in $3 \mathrm{~mL}$ hexane and centrifuged at $6500 \mathrm{rpm}$ (5668 $\mathrm{g})$ for $7 \mathrm{~min}$ to separate the stable core/shell NPLs in the supernatant from aggregated core/shell NPLs. The core/shell NPLs in the supernatant were then filtered using a $0.2 \mu \mathrm{m}$ pore-size polytetrafluoroethylene (PTFE) membrane filter and stored in the dark under ambient conditions.

The above synthesis can be performed using different ratios of $\mathrm{Cd}(\text { oleate })_{2}$ to $\mathrm{Zn}$ (oleate) $)_{2}$ keeping the added amount of metal oleate constant. In general, a higher $\mathrm{Zn}$ :Cd ratio leads to a slight improvement of the colloidal stability of the final core/shell NPLs. For pure Cd(oleate)2, CdS is formed as a side product. ${ }^{25}$ This side product can be efficiently removed by increasing the amount of hexane added upon cooling of the reaction product and the subsequent purification steps.

The quality of the resulting core/shell nanoplatelets is improved if the temperature ramp from $80{ }^{\circ} \mathrm{C}$ to the reaction temperature of $300{ }^{\circ} \mathrm{C}$ is slowed down at around $270{ }^{\circ} \mathrm{C}$, and reaches $300{ }^{\circ} \mathrm{C}$ after $\sim 30 \mathrm{~min}$.

Synthesis of CdSe/CdS/ZnS Core/Shell/Shell NPLs. In a typical synthesis, 4-monolayer CdSe NPLs [an amount equivalent to $1 \mathrm{~mL}$ of NPL dispersion with an optical density (OD) of 120 at the 
lowest energy excitonic peak, assuming a 1-cm optical path length], $10 \mathrm{~mL}$ ODE, $400 \mu \mathrm{L}$ OA, and $270 \mathrm{mg} \mathrm{Cd}(\text { oleate })_{2}(0.4 \mathrm{mmol})$ were added to a $100-\mathrm{mL}$ round-bottom flask. The mixture was degassed under vacuum for $35 \mathrm{~min}$ at $\mathrm{RT}$ and $15 \mathrm{~min}$ at $80^{\circ} \mathrm{C}$. Afterwards, $2 \mathrm{~mL} \mathrm{OAm}$ (stored in a $\mathrm{N}_{2}$-filled glovebox) was added and the temperature was raised to $300{ }^{\circ} \mathrm{C}$ under $\mathrm{N}_{2}$ at a rate of $\sim 15{ }^{\circ} \mathrm{C} / \mathrm{min}$. Starting at $165^{\circ} \mathrm{C}$, a solution of $83 \mu \mathrm{L}$ 1-octanethiol $(0.48 \mathrm{mmol})$ dissolved in $9 \mathrm{~mL}$ ODE was injected at a rate of $4.5 \mathrm{~mL} / \mathrm{h}$. After complete injection, the solution was cooled to $200{ }^{\circ} \mathrm{C}, 2 \mathrm{~mL}$ OA (stored in a $\mathrm{N}_{2}$-filled glovebox) was added, and the mixture was kept at $200{ }^{\circ} \mathrm{C}$ for $40 \mathrm{~min}$. Then, the mixture was cooled to $120^{\circ} \mathrm{C}$ and degassed under vacuum for $30 \mathrm{~min}$. Afterwards, the temperature was raised to $280{ }^{\circ} \mathrm{C}$ under $\mathrm{N}_{2}$ at a rate of $\sim 15{ }^{\circ} \mathrm{C} / \mathrm{min}$. Starting at $200{ }^{\circ} \mathrm{C}$, a solution of $34.7 \mu \mathrm{L} 1$ octanethiol $(0.2 \mathrm{mmol})$ dissolved in $3 \mathrm{~mL}$ ODE and a solution of $0.5 \mathrm{~mL} \mathrm{Zn}(\mathrm{OA})_{2}(0.2 \mathrm{M})$ dissolved in $3 \mathrm{~mL}$ ODE were simultaneously injected at a rate of $3 \mathrm{~mL} / \mathrm{h}$. After complete injection, $1 \mathrm{~mL} \mathrm{OA}$ (stored in a $\mathrm{N}_{2}$-filled glovebox) was injected and the product was cooled down, and, at $40{ }^{\circ} \mathrm{C}, \sim 5 \mathrm{~mL}$ hexane was added. The core/shell NPLs were purified by selective precipitation as described for 4monolayer-CdSe/Cdx $Z_{1-x} \mathrm{~S}$. The purified core/shell NPLs were stored in the dark under ambient conditions.

Synthesis of CdSe/ZnS Core/Shell NPLs with a Smooth Shell. In a typical synthesis, 4monolayer CdSe NPLs [an amount equivalent to $1 \mathrm{~mL}$ of NPL dispersion with an optical density (OD) of 60 at the lowest energy excitonic peak, assuming a 1-cm optical path length], $5 \mathrm{~mL}$ ODE, $200 \mu \mathrm{L}$ $\mathrm{OA}$, and $31.4 \mathrm{mg} \mathrm{Zn(oleate)})_{2}(0.05 \mathrm{mmol})$ were added to a $50-\mathrm{mL}$ round-bottom flask. The mixture was degassed under vacuum for $35 \mathrm{~min}$ at $\mathrm{RT}$ and $15 \mathrm{~min}$ at $80{ }^{\circ} \mathrm{C}$. Afterwards, $1 \mathrm{~mL}$ OAm (stored in a $\mathrm{N}_{2}$-filled glovebox) was added and the temperature was raised to $300{ }^{\circ} \mathrm{C}$ under $\mathrm{N}_{2}$ at a rate of $\sim 15^{\circ} \mathrm{C} / \mathrm{min}$. Starting at $165{ }^{\circ} \mathrm{C}$, a solution of $42 \mu \mathrm{L} 1$-octanethiol $(0.24 \mathrm{mmol})$ dissolved in $4 \mathrm{~mL}$ ODE and $2 \mathrm{~mL} \mathrm{OA}$, and a solution of $0.75 \mathrm{~mL} \mathrm{Zn}(\mathrm{OA})_{2}(0.2 \mathrm{M})$ dissolved in $6 \mathrm{~mL}$ ODE were injected simultaneously at a rate of $3 \mathrm{~mL} / \mathrm{h}$. After complete injection, the solution was kept at $300{ }^{\circ} \mathrm{C}$ for $30 \mathrm{~min}$. Subsequently, the product was cooled down, and, at $40{ }^{\circ} \mathrm{C}, \sim 2 \mathrm{~mL}$ hexane was added. The core/shell NPLs were purified by selective precipitation. In a first step, $5 \mathrm{~mL}$ methyl acetate was added and the mixture was centrifuged at $6500 \mathrm{rpm}(5668 \mathrm{~g})$ for $10 \mathrm{~min}$. The core/shell NPLs in the 
precipitate were re-dispersed in $4 \mathrm{~mL}$ hexane. [If a significant amount of core/shell NPLs remained in the supernatant after this centrifugation step, they can be precipitated by adding methyl acetate and centrifuging at $6500 \mathrm{rpm}(5668 \mathrm{~g})$.] Then, the dispersion was centrifuged at $6500 \mathrm{rpm}(5668 \mathrm{~g})$ for $10 \mathrm{~min}$ to remove side products and unreacted precursors. The core/shell NPLs in the supernatant were then precipitated from the hexane dispersion by adding $\sim 5 \mathrm{~mL}$ methyl acetate and centrifuging at $6500 \mathrm{rpm}(5668 \mathrm{~g})$ for $10 \mathrm{~min}$. This precipitation process was repeated. The core/shell NPLs were then re-dispersed in $3 \mathrm{~mL}$ hexane and centrifuged at $6500 \mathrm{rpm}(5668 \mathrm{~g})$ for $7 \mathrm{~min}$ to separate the stable core/shell NPLs in the supernatant from aggregated core/shell NPLs. The core/shell NPLs in the supernatant were then filtered using a $0.2 \mu \mathrm{m}$ pore-size PTFE filter and stored in the dark under ambient conditions.

Synthesis of CdSe/ZnSe Core/Shell NPLs. In a typical synthesis, 4-monolayer CdSe NPLs [an amount equivalent to $1 \mathrm{~mL}$ of NPL dispersion with an optical density (OD) of 60 at the lowest energy excitonic peak, assuming a 1-cm optical path length], $5 \mathrm{~mL}$ ODE, $200 \mu \mathrm{L}$ OA, and $62.8 \mathrm{mg}$ $\mathrm{Zn}$ (oleate)2 $(0.1 \mathrm{mmol})$ were added to a $50-\mathrm{mL}$ round-bottom flask. The mixture was degassed under vacuum for $35 \mathrm{~min}$ at $\mathrm{RT}$ and $15 \mathrm{~min}$ at $80^{\circ} \mathrm{C}$. Afterwards, $1 \mathrm{~mL} \mathrm{OAm}$ (stored in a $\mathrm{N}_{2}$-filled glovebox) was added and the temperature was raised to $300{ }^{\circ} \mathrm{C}$ under $\mathrm{N}_{2}$ at a rate of $\sim 15^{\circ} \mathrm{C} / \mathrm{min}$. Starting at $165{ }^{\circ} \mathrm{C}$, a solution of $1 \mathrm{~mL}$ Se-ODE stock solution dissolved in $3 \mathrm{~mL}$ ODE and $2 \mathrm{~mL} \mathrm{OA}$ was injected at a rate of $3 \mathrm{~mL} / \mathrm{h}$. After complete injection, the solution was kept at $300{ }^{\circ} \mathrm{C}$ for $30 \mathrm{~min}$. Subsequently, the product was cooled down, and, at $50{ }^{\circ} \mathrm{C}, \sim 2 \mathrm{~mL}$ hexane was added. The core/shell NPLs were purified by selective precipitation. In a first step, methyl acetate was added until the mixture turned turbid, and it was centrifuged at $6500 \mathrm{rpm}$ (5668 g) for $10 \mathrm{~min}$. The core/shell NPLs in the precipitate were re-dispersed in $4 \mathrm{~mL}$ hexane and precipitated by adding $\sim 5 \mathrm{~mL}$ methyl acetate and centrifuging at $6500 \mathrm{rpm}(5668 \mathrm{~g})$ for $10 \mathrm{~min}$. This precipitation process was repeated. The core/shell NPLs were then re-dispersed in $3 \mathrm{~mL}$ hexane and centrifuged at $6500 \mathrm{rpm}(5668 \mathrm{~g})$ for $7 \mathrm{~min}$ to separate the stable core/shell NPLs in the supernatant from aggregated core/shell NPLs. The core/shell NPLs in the supernatant were then filtered using a $0.2 \mu \mathrm{m}$ pore-size PTFE filter and stored in the dark under ambient conditions. 
Synthesis of CdSe/Cdx $\mathrm{Zn}_{1-\mathrm{x}} \mathrm{S}$ Core/Shell NPLs with 6-Monolayer-Thick CdSe NPL Cores.

In a typical synthesis, 6-monolayer CdSe NPLs [an amount equivalent to $1 \mathrm{~mL}$ of NPL dispersion with an optical density (OD) of 20 at the lowest energy excitonic peak, assuming a 1-cm optical path length], $5 \mathrm{~mL}$ ODE, $200 \mu \mathrm{L} \mathrm{OA}, 45 \mathrm{mg}$ Cd(oleate)2, and $83.8 \mathrm{mg} \mathrm{Zn}$ (oleate)2 (total of $0.2 \mathrm{mmol}$ metal oleate, molar ratio of 1:2) were added to a 50 -mL round-bottom flask. The mixture was degassed under vacuum for $35 \mathrm{~min}$ at $\mathrm{RT}$ and $15 \mathrm{~min}$ at $80^{\circ} \mathrm{C}$. Afterwards, $1 \mathrm{~mL} \mathrm{OAm}$ (stored in a $\mathrm{N}_{2}$-filled glovebox) was added and the temperature was raised to $300{ }^{\circ} \mathrm{C}$ under $\mathrm{N}_{2}$ at a rate of $\sim 15{ }^{\circ} \mathrm{C} / \mathrm{min}$. Starting at $165^{\circ} \mathrm{C}$, a solution of $42 \mu \mathrm{L}$ 1-octanethiol $(0.24 \mathrm{mmol})$ dissolved in $3.5 \mathrm{~mL}$ ODE and $1 \mathrm{~mL}$ OA was injected at a rate of $2.25 \mathrm{~mL} / \mathrm{h}$. After complete injection, the solution was kept at $300{ }^{\circ} \mathrm{C}$ for $40 \mathrm{~min}$. Subsequently, the product was cooled down, and, at $40{ }^{\circ} \mathrm{C}, \sim 2.5 \mathrm{~mL}$ hexane was added. The core/shell NPLs were purified by selective precipitation. In a first step, the mixture was centrifuged at $6500 \mathrm{rpm}$ (5668 g) for $6 \mathrm{~min}$. The core/shell NPLs in the precipitate were re-dispersed in $3 \mathrm{~mL}$ hexane. [If a significant amount of core/shell NPLs remained in the supernatant after this centrifugation step, they can be precipitated by adding methyl acetate and centrifuging at $6500 \mathrm{rpm}(5668 \mathrm{~g})$.] Then, $\sim 3 \mathrm{~mL}$ methyl acetate was added to the dispersion of core/shell NPLs in hexane until the mixture turned turbid, and it was centrifuged at $6500 \mathrm{rpm}$ (5668 g) for $10 \mathrm{~min}$. This process was repeated. The core/shell NPLs were then re-dispersed in $2 \mathrm{~mL}$ hexane and centrifuged at $6500 \mathrm{rpm}$ (5668 $\mathrm{g}$ ) for 7 min to separate the stable core/shell NPLs in the supernatant from aggregated core/shell NPLs. The core/shell NPLs in the supernatant were then filtered using a $0.2 \mu \mathrm{m}$ pore-size PTFE filter and stored in the dark under ambient conditions.

Optimization of CdSe/Cdx $Z_{n_{1-x}} S$ Core/Shell NPLs. Reactions were performed by mixing 4monolayer CdSe NPLs [an amount equivalent to $1 \mathrm{~mL}$ of NPL dispersion with an optical density (OD) of 60 at the lowest energy excitonic peak, assuming a $1-\mathrm{cm}$ optical path length], $5 \mathrm{~mL}$ ODE, and $200 \mu \mathrm{L}$ OA together with various molar ratios of $\mathrm{Cd}(\text { oleate })_{2}$ and $\mathrm{Zn}$ (oleate)2, respectively, in a 50 $\mathrm{mL}$ round-bottom flask. Reactions were performed with a total metal oleate concentration of $0.05,0.1$, and $0.2 \mathrm{mmol}$. The mixture was then degassed under vacuum for $35 \mathrm{~min}$ at $\mathrm{RT}$ and $15 \mathrm{~min}$ at $80{ }^{\circ} \mathrm{C}$. Afterwards, $1 \mathrm{~mL}$ OAm (stored in a $\mathrm{N}_{2}$-filled glovebox) was added and the temperature was raised to 
$300{ }^{\circ} \mathrm{C}$ under $\mathrm{N}_{2}$ at a rate of $\sim 15^{\circ} \mathrm{C} / \mathrm{min}$. Starting at $165^{\circ} \mathrm{C}$, a solution of 1 -octanethiol dissolved in ODE and OA was slowly injected. For reactions with $0.05 \mathrm{mmol}$ metal oleate, $0.06 \mathrm{mmol} 1$ octanethiol was dissolved in $1.3 \mathrm{~mL}$ ODE and $0.7 \mathrm{~mL}$ OA. For reactions with $0.1 \mathrm{mmol}$ metal oleate, $0.12 \mathrm{mmol}$ 1-octanethiol was dissolved in $2 \mathrm{~mL}$ ODE and $1 \mathrm{~mL}$ OA. For reactions with $0.2 \mathrm{mmol}$ metal oleate, $0.24 \mathrm{mmol} 1$-octanethiol was dissolved in $3.5 \mathrm{~mL} \mathrm{ODE}$ and $1 \mathrm{~mL}$ OA. The injection rate of 1-octanethiol was $0.12 \mathrm{mmol} / \mathrm{h}$ in all experiments. After complete injection, the solution was kept at $300{ }^{\circ} \mathrm{C}$ for $30 \mathrm{~min}$. Subsequently, the product was cooled down and, at $40{ }^{\circ} \mathrm{C}, \sim 2 \mathrm{~mL}$ hexane was added. The core/shell NPLs were purified by selective precipitation as described in the synthesis for $\mathrm{CdSe} / \mathrm{Cd}_{\mathrm{x}} \mathrm{Zn}_{1-\mathrm{x}} \mathrm{S}$ NPLs.

Heating of CdSe Core NPLs in the Presence of Metal Oleates. For the etching experiments, 4monolayer CdSe NPLs [an amount equivalent to $1 \mathrm{~mL}$ of NPL dispersion with an optical density (OD) of 60 at the lowest energy excitonic peak, assuming a 1-cm optical path length], $5 \mathrm{~mL}$ ODE, and $200 \mu \mathrm{L}$ OA were mixed together with $135 \mathrm{mg} \mathrm{Cd}$ (oleate) $)_{2}(0.2 \mathrm{mmol}), 125.7 \mathrm{mg} \mathrm{Zn}$ (oleate) (0.2 mmol), or $31.4 \mathrm{mg} \mathrm{Zn}$ (oleate) $2(0.05 \mathrm{mmol})$ in a $50-\mathrm{mL}$ round-bottom flask. The mixture was then degassed under vacuum for $35 \mathrm{~min}$ at $\mathrm{RT}$ and $15 \mathrm{~min}$ at $80^{\circ} \mathrm{C}$. Then, $1 \mathrm{~mL} \mathrm{OAm}$ (stored in a $\mathrm{N}_{2-}$ filled glovebox) was added. Afterwards, the temperature was raised to $300{ }^{\circ} \mathrm{C}$ under $\mathrm{N}_{2}$ at a rate of $\sim 15^{\circ} \mathrm{C} / \mathrm{min}$, and aliquots were taken at different temperatures to measure the evolution of the absorbance, the size, and the shape. The same batch of CdSe NPLs was used for all experiments to ensure identical starting conditions. To enable quantitative comparisons, all optical absorption measurements were performed by diluting $40 \mu \mathrm{L}$ of the sample in $1960 \mu \mathrm{L}$ hexane and measured in a 1-cm path-length quartz cuvette.

Fabrication of Core/Shell NPL Ring Resonators. NPL ring resonators were fabricated using the template-stripping technique from Prins et al. ${ }^{55}$ to obtain ring structures as described by le Feber et al. ${ }^{54}$ In a first step, silicon (100) templates were patterned using electron-beam lithography (Vistec, EBGP 520) and subsequent inductive plasma deep reactive-ion etching (Oxford, Plasmalab System 100). To facilitate stripping of the NPL films, the silicon templates were functionalized with a selfassembled monolayer of octadecyltrichlorosilane. Uniform films of NPLs were then deposited by 
drop-casting $35 \mu \mathrm{L}$ of NPL dispersions in a hexane:octane mixture of 9:1. Template stripping was then performed by attaching a glass slide to the deposited NPL film with an epoxy glue (Norland Products, NOA 61) and curing it under a 365-nm ultraviolet lamp. The NPL-film/epoxy/glass stack was then removed from the silicon template by stripping it off with a razor blade. Subsequently, the Si template was cleaned by ultra-sonication in chloroform for $5 \mathrm{~min}$ for re-use.

Absorbance. Optical absorption spectra for ultraviolet to visible wavelengths (UV-Vis) were collected using a Varian Cary 50 spectrometer. The measurements were performed by diluting the samples in hexane and using 1-cm path-length quartz cuvettes.

Photoluminescence (PL) and Photoluminescence Decays. PL spectra were recorded at RT using an Edinburgh Instruments FLS 980 fluorometer. For the standard PL spectra presented in this work, the excitation wavelength was set to $400 \mathrm{~nm}$. The excitation-emission maps shown in Figure 6 were recorded using excitation wavelengths spaced by $5 \mathrm{~nm}$. For photoluminescence decays, the photoluminescence-lifetime capability of the same fluorometer was used. A pulsed laser diode at $373 \mathrm{~nm}$ with a repetition rate of $100 \mathrm{kHz}$ was used as the excitation source. The detected wavelength was set to the peak emission wavelength for the various core- and core/shell NPLs. The detector was the same cooled Hamamatsu R928P photomultiplier tube used to collect the PL results.

Photoluminescence Quantum Yield (QY). QY values were measured at RT using a Hamamatsu C11347 Quantaurus-QY spectrometer equipped with an integrating sphere. QY values were obtained for different excitation wavelengths from $370 \mathrm{~nm}$ to the emission onset in $10-\mathrm{nm}$ steps or by performing single measurements exciting the samples at $400 \mathrm{~nm}$. Unless stated, single QY values reported in the main text and the SI are obtained by exciting at $400 \mathrm{~nm}$. The measurements were performed after NPL purification by diluting the samples in hexane and using quartz cuvettes with a 1-cm path length. The cuvettes were cleaned in a $1 \%$-solution of Hellmanex ${ }^{\mathrm{TM}}$ III after QY measurements to ensure accurate results.

Transmission Electron Microscopy (TEM), Scanning Transmission Electron Microscopy (STEM), and Energy Dispersive X-ray Spectroscopy (EDS). TEM, STEM, and EDS were 
performed using an FEI Talos F200X electron microscope operated at $200 \mathrm{kV}$. The samples were prepared by drop-casting nanocrystals dispersed in hexane on carbon-coated copper grids.

Atomic-resolution images were acquired with a JEOL JEM-ARM300F Grand ARM microscope operated at $300 \mathrm{kV}$. The sample was prepared by drop-casting nanocrystals dispersed in hexane on silicon dioxide support films.

Powder X-ray Diffraction (XRD). XRD measurements were performed using a Bruker D2 Phaser instrument $\left(30 \mathrm{kV}, 10 \mathrm{~mA}, \lambda_{\mathrm{CuK} \alpha}=1.54184 \AA\right.$ ) $)$. The samples were drop-casted from highly concentrated hexane dispersions onto zero-background Si holders.

Rutherford Backscattering Spectrometry (RBS). RBS was performed at the ETH Laboratory of Ion Beam Physics. The samples were prepared by spin-coating NPL dispersions onto Si(100) substrates. Spectra were acquired using a $2 \mathrm{MeV}{ }^{4} \mathrm{He}$ beam and a silicon PIN diode detector at $\theta=168^{\circ}$. The collected RBS data was analyzed using simulations by the RUMP code. ${ }^{57}$

Nanoplatelet Size and Shape Evaluation. For the analysis presented in Figure S7, HAADF STEM images of NPLs from aliquots of the etching experiments were analyzed using a Python script. Standard image-processing algorithms were taken from the image processing library scikit-image. For the size and shape analysis, each NPL was extracted individually from the image as displayed in Figure S14. To separate NPLs that are touching, a watershed algorithm was applied recursively to assemblies of area larger than $200 \mathrm{~nm}^{2}$. At the end of the process, particles that were at the image edge, aggregated, stacked, or otherwise could not be separated by the watershed algorithm were eliminated from the dataset. All evaluated images underwent a visual check, and segmented contours that contained zero, multiple, or only partial NPLs were manually cleared from the dataset. Less than 5\% of the contours contained falsely detected NPLs.

In total, 37 images of NPLs at a magnification of $320,000 \mathrm{X}$ were evaluated with the same script and the same set of parameters. The number of detected NPLs ranged between 417 and 921 for the different aliquots. A random sample of 400 NPLs was chosen from each aliquot to obtain statistics for the lateral area, and the lateral area normalized by the convex hull area. Boxplots of random samples of 100 and 200 NPLs displayed the same trend as the boxplot for the 400-NPL sample. 
Nanoplatelet Ring Lasers. Laser experiments were performed using a 405-nm pulsed excitation ( $\sim 340$ fs pulse duration, $10 \mathrm{kHz}$ repetition rate), generated by a collinear optical parametric amplifier (Spectra-Physics, Spirit OPA) pumped by a 1040-nm laser (Spectra-Physics, Spirit 1040-8). To detect the emission signal, an inverted microscope (Nikon, Eclipse Ti-U) with a 50x air objective (Nikon, 0.8 numerical aperture) was used. The beam was directed to the sample through a dichroic beamsplitter (488-nm long pass, AHV, Analysentechnik). The emission was collected by the same objective and directed through a 503-nm long-pass emission filter (AHF, Analysentechnik) into a spectrometer (Andor, Shamrock 303i). Spectra were obtained by dispersing the emission with a $300 \mathrm{lines} / \mathrm{mm}$ grating (500 nm blaze), and detecting the output with a complementary metal oxide semiconductor (CMOS) camera (Andor, Zyla 4.2 Plus). The measurements were performed under ambient conditions and analyzed as reported by le Feber et al. ${ }^{54}$

\section{ASSOCIATED CONTENT}

\section{Supporting Information}

The Supporting Information is available free of charge on the ACS Publications website at DOI: $10.1021 /$

A description of the optimization of the synthesis of CdSe/ZnS core/shell NPLs, Table S1, and

Figures S1-S14, providing extracted optical parameters (emission peak wavelengths, linewidths, QYs, and lifetime values), additional optical spectra, electron micrographs, EDS, RBS, laser power curves, and the NPL size/shape analysis.

\section{AUTHOR INFORMATION}

\section{Corresponding Author}

*Email: dnorris@ethz.ch.

\section{ORCID}

Aurelio A. Rossinelli: 0000-0001-6930-4190

Henar Rojo: 0000-0003-1543-6264

Aniket S. Mule: 0000-0001-8387-080X 
Marianne Aellen: 0000-0003-1548-0433

Ario Cocina: 0000-0003-1560-5849

Eva De Leo: 0000-0002-9677-0274

Robin Schäublin: 0000-0002-8379-9705

David J. Norris: 0000-0002-3765-0678

\section{Notes}

The authors declare no competing financial interest.

\section{ACKNOWLEDGMENTS}

This work was supported by the Swiss National Science Foundation under Grant No. 200021-165559, and the European Research Council under the European Union's Seventh Framework Program (FP/2007-2013) / ERC Grant Agreement No. 339905 (QuaDoPS Advanced Grant). We thank M. Döbeli and S. Pratsinis for assistance with RBS and XRD, respectively, P. Guyot-Sionnest, F. Knüsel, S. Mazzotti, and M. Wörle for stimulating discussions, A. Riedinger and P. Kumar for reading the manuscript, and S. Meyer for technical assistance. We utilized facilities at the Scientific Center for Optical and Electronic Microscopy (ScopeM) and the Laboratory of Ion Beam Physics at ETH Zurich.

\section{REFERENCES}

1. J. Owen; L. Brus, Chemical synthesis and luminescence applications of colloidal semiconductor quantum dots. J. Am. Chem. Soc. 2017, 139, 10939-10943.

2. Y. Shirasaki; G. J. Supran; M. G. Bawendi; V. Bulović, Emergence of colloidal quantum-dot light-emitting technologies. Nat. Photon. 2012, 7, 13-23.

3. Y. E. Panfil; M. Oded; U. Banin, Colloidal quantum nanostructures: Emerging materials for display applications. Angew. Chem. Int. Ed. 2018, 57, 4274-4295.

4. J. Lim; Y.-S. Park; V. I. Klimov, Optical gain in colloidal quantum dots achieved with directcurrent electrical pumping. Nat. Mater. 2018, 17, 42-49.

5. Y. Wang; I. Fedin; H. Zhang; D. V. Talapin, Direct optical lithography of functional inorganic nanomaterials. Science 2017, 357, 385-388. 
6. P. Reiss; M. Protière; L. Li, Core/shell semiconductor nanocrystals. Small 2009, 5, 154-168.

7. S. Ithurria; B. Dubertret, Quasi 2D colloidal CdSe platelets with thicknesses controlled at the atomic level. J. Am. Chem. Soc. 2008, 130, 16504-16505.

8. A. Riedinger; F. D. Ott; A. Mule; S. Mazzotti; P. N. Knüsel; S. J. P. Kress; F. Prins; S. C. Erwin; D. J. Norris, An intrinsic growth instability in isotropic materials leads to quasi-two-dimensional nanoplatelets. Nat. Mater. 2017, 16, 743-748.

9. S. Ithurria; M. D. Tessier; B. Mahler; R. P. S. M. Lobo; B. Dubertret; A. L. Efros, Colloidal nanoplatelets with two-dimensional electronic structure. Nat. Mater. 2011, 10, 936-941.

10. F. Rajadell; J. I. Climente; J. Planelles, Excitons in core-only, core-shell and core-crown CdSe nanoplatelets: Interplay between in-plane electron-hole correlation, spatial confinement, and dielectric confinement. Phys. Rev. B 2017, 96, 035307.

11. M. Pelton, Carrier dynamics, optical gain, and lasing with colloidal quantum wells. J. Phys. Chem. C 2018, 122, 10659-10674.

12. F. Zhang; S. Wang; L. Wang; Q. Lin; H. Shen; W. Cao; C. Yang; H. Wang; L. Yu; Z. Du; J. Xue; L. S. Li, Super color purity green quantum dot light-emitting diodes fabricated by using $\mathrm{CdSe} / \mathrm{CdS}$ nanoplatelets. Nanoscale 2016, 8, 12182-12188.

13. S. J. Lim; W. Kim; S. K. Shin, Surface-dependent, ligand-mediated photochemical etching of CdSe nanoplatelets. J. Am. Chem. Soc. 2012, 134, 7576-7579.

14. M. Olutas; B. Guzelturk; Y. Kelestemur; A. Yeltik; S. Delikanli; H. V. Demir, Lateral sizedependent spontaneous and stimulated emission properties in colloidal CdSe nanoplatelets. $A C S$ Nano 2015, 9, 5041-5050.

15. S. Singh; R. Tomar; S. ten Brinck; J. De Roo; P. Geiregat; J. C. Martins; I. Infante; Z. Hens, Colloidal CdSe nanoplatelets, a model for surface chemistry/optoelectronic property relations in semiconductor canocrystals. J. Am. Chem. Soc. 2018, 140, 13292-13300.

16. O. Chen; J. Zhao; V. P. Chauhan; J. Cui; C. Wong; D. K. Harris; H. Wei; H.-S. Han; D. Fukumura; R. K. Jain; M. G. Bawendi, Compact high-quality CdSe-CdS core-shell nanocrystals with narrow emission linewidths and suppressed blinking. Nat. Mater. 2013, 12, 445-451. 
17. K. Boldt; N. Kirkwood; G. A. Beane; P. Mulvaney, Synthesis of highly luminescent and photostable, graded shell $\mathrm{CdSe} / \mathrm{Cd}_{\mathrm{x}} \mathrm{Zn}_{1-\mathrm{x}} \mathrm{S}$ nanoparticles by in situ alloying. Chem. Mater. 2013, 25, $4731-4738$.

18. Y. Wang; K. E. Fong; S. Yang; Van D. Ta; Y. Gao; Z. Wang; V. Nalla; H. V. Demir; H. Sun, Unraveling the ultralow threshold stimulated emission from $\mathrm{CdZnS} / \mathrm{ZnS}$ quantum dot and enabling high-Q microlasers. Laser Photonics Rev. 2015, 9, 507-516.

19. F. D. Ott; A. Riedinger; D. R. Ochsenbein; P. N. Knüsel; S. C. Erwin; M. Mazzotti; D. J. Norris, Ripening of semiconductor nanoplatelets. Nano Lett. 2017, 17, 6870-6877.

20. S. Ithurria; D. V. Talapin, Colloidal atomic layer deposition (c-ALD) using self-limiting reactions at nanocrystal surface coupled to phase transfer between polar and nonpolar media. $J$. Am. Chem. Soc. 2012, 134, 18585-18590.

21. Y. Kelestemur; B. Guzelturk; O. Erdem; M. Olutas; K. Gungor; H. V. Demir, Platelet-in-box colloidal quantum wells: CdSe/CdS@CdS core/crown@shell heteronanoplatelets. Adv. Funct. Mater. 2016, 26, 3570-3579.

22. B. Mahler; B. Nadal; C. Bouet; G. Patriarche; B. Dubertret, Core/shell colloidal semiconductor nanoplatelets. J. Am. Chem. Soc. 2012, 134, 18591-18598.

23. M. D. Tessier; B. Mahler; B. Nadal; H. Heuclin; S. Pedetti; B. Dubertret, Spectroscopy of colloidal semiconductor core/shell nanoplatelets with high quantum yield. Nano Lett. 2013, 13, $3321-3328$.

24. A. Polovitsyn; Z. Dang; J. L. Movilla; B. Martín-García; A. H. Khan; G. H. V. Bertrand; R. Brescia; I. Moreels, Synthesis of air-stable CdSe/ZnS core-shell nanoplatelets with tunable emission wavelength. Chem. Mater. 2017, 29, 5671-5680.

25. A. A. Rossinelli; A. Riedinger; P. Marqués-Gallego; P. N. Knüsel; F. V. Antolinez; D. J. Norris, High-temperature growth of thick-shell $\mathrm{CdSe} / \mathrm{CdS}$ core/shell nanoplatelets. Chem. Commun. 2017, 53, 9938-9941.

26. M. A. Hines; P. Guyot-Sionnest, Synthesis and characterization of strongly luminescing ZnScapped CdSe nanocrystals. J. Phys. Chem. 1996, 100, 468-471. 
27. X. Peng; M. C. Schlamp; A. V. Kadavanich; A. P. Alivisatos, Epitaxial growth of highly luminescent $\mathrm{CdSe} / \mathrm{CdS}$ core/shell nanocrystals with photostability and electronic accessibility. J. Am. Chem. Soc. 1997, 119, 7019-7029.

28. B. O. Dabbousi; J. Rodriguez-Viejo; F. V. Mikulec; J. R. Heine; H. Mattoussi; R. Ober; K. F. Jensen; M. G. Bawendi, (CdSe)ZnS core--shell quantum dots: Synthesis and characterization of a size series of highly luminescent nanocrystallites. J. Phys. Chem. B 1997, 101, 9463-9475.

29. H. Cruguel; C. Livache; B. Martinez; S. Pedetti; D. Pierucci; E. Izquierdo; M. Dufour; S. Ithurria; H. Aubin; A. Ouerghi; E. Lacaze; M. G. Silly; B. Dubertret; E. Lhuillier, Electronic structure of CdSe-ZnS 2D nanoplatelets. Appl. Phys. Lett. 2017, 110, 152103.

30. Y. Altintas; U. Quliyeva; K. Gungor; O. Erdem; Y. Kelestemur; E. Mutlugun; M. V. Kovalenko; H. V. Demir, Highly stable, near-unity efficiency atomically flat semiconductor nanocrystals of $\mathrm{CdSe} / \mathrm{ZnS}$ hetero-nanoplatelets enabled by ZnS-shell hot-injection growth. Small 2019, 15, 1804854.

31. Z.-J. Jiang; D. F. Kelley, Stranski-Krastanov shell growth in ZnTe/CdSe core/shell nanocrystals. J. Phys. Chem. C 2013, 117, 6826-6834.

32. S. G. Kwon; G. Krylova; P. J. Phillips; R. F. Klie; S. Chattopadhyay; T. Shibata; E. E. Bunel; Y. Liu; V. B. Prakapenka; B. Lee; E. V. Shevchenko, Heterogeneous nucleation and shape transformation of multicomponent metallic nanostructures. Nat. Mater. 2015, 14, 215-223.

33. B. Ji; Y. E. Panfil; N. Waiskopf; S. Remennik; I. Popov; U. Banin, Strain-controlled shell morphology on quantum rods. Nat. Commun. 2019, 10, 2.

34. N. Oh; M. Shim, Metal oleate induced etching and growth of semiconductor nanocrystals, nanorods, and their heterostructures. J. Am. Chem. Soc. 2016, 138, 10444-10451.

35. R. Xie; U. Kolb; J. Li; T. Basché; A. Mews, Synthesis and characterization of highly luminescent CdSe-Core CdS/Zn ${ }_{0.5} \mathrm{Cd}_{0.5} \mathrm{~S} / \mathrm{ZnS}$ multishell nanocrystals. J. Am. Chem. Soc. 2005, 127, 74807488.

36. L. Manna; E. C. Scher; L.-S. Li; A. P. Alivisatos, Epitaxial growth and photochemical annealing of graded CdS/ZnS shells on colloidal CdSe nanorods. J. Am. Chem. Soc. 2002, 124, 7136-7145. 
37. I. Nakonechnyi; M. Sluydts; Y. Justo; J. Jasieniak; Z. Hens, Mechanistic insights in seeded growth synthesis of colloidal core/shell quantum dots. Chem. Mater. 2017, 29, 4719-4727.

38. S. Abe; R. K. Capek; B. De Geyter; Z. Hens, Reaction chemistry/nanocrystal property relations in the hot injection synthesis, the role of the solute solubility. ACS Nano 2013, 7, 943-949.

39. D. Shaw, Diffusion mechanisms in II-VI materials. J. Cryst. Growth 1988, 86, 778-796.

40. B. M. Saidzhonov; V. F. Kozlovsky; V. B. Zaytsev; R. B. Vasiliev, Ultrathin CdSe/CdS and $\mathrm{CdSe} / \mathrm{ZnS}$ core-shell nanoplatelets: The impact of the shell material on the structure and optical properties. J. Lumin. 2019, 209, 170-178.

41. J. Cui; A. P. Beyler; I. Coropceanu; L. Cleary; T. R. Avila; Y. Chen; J. M. Cordero; S. L. Heathcote; D. K. Harris; O. Chen; J. Cao; M. G. Bawendi, Evolution of the single-nanocrystal photoluminescence linewidth with size and shell: implications for exciton-phonon coupling and the optimization of spectral linewidths. Nano Lett. 2016, 16, 289-296.

42. N. Tschirner; H. Lange; A. Schliwa; A. Biermann; C. Thomsen; K. Lambert; R. Gomes; Z. Hens, Interfacial alloying in $\mathrm{CdSe} / \mathrm{CdS}$ heteronanocrystals: a raman spectroscopy analysis. Chem. Mater. 2012, 24, 311-318.

43. A. Rubin-Brusilovski; Y. Jang; A. Shapiro; A. Safran; A. Sashchiuk; E. Lifshitz, Influence of interfacial strain on optical properties of $\mathrm{PbSe} / \mathrm{PbS}$ colloidal quantum dots. Chem. Mater. 2016, 28, 9056-9063.

44. I. Coropceanu; A. Rossinelli; J. R. Caram; F. S. Freyria; M. G. Bawendi, Slow-injection growth of seeded $\mathrm{CdSe} / \mathrm{CdS}$ nanorods with unity fluorescence quantum yield and complete shell to core energy transfer. ACS Nano 2016, 10, 3295-3301.

45. S. Christodoulou; J. I. Climente; J. Planelles; R. Brescia; M. Prato; B. Martín-García; A. H. Khan; I. Moreels, Chloride-induced thickness control in CdSe nanoplatelets. Nano Lett. 2018, 18, 6248-6254.

46. W. Cho; S. Kim; I. Coropceanu; V. Srivastava; B. T. Diroll; A. Hazarika; I. Fedin; G. Galli; R. D. Schaller; D. V. Talapin, Direct synthesis of six-monolayer $(1.9 \mathrm{~nm})$ thick zinc-blende CdSe nanoplatelets emitting at $585 \mathrm{~nm}$. Chem. Mater. 2018, 30, 6957-6960. 
47. V. I. Klimov; A. A. Mikhailovsky; D. W. McBranch; C. A. Leatherdale; M. G. Bawendi, Quantization of multiparticle auger rates in semiconductor quantum dots. Science 2000, 287, 1011-1013.

48. G. E. Cragg; A. L. Efros, Suppression of auger processes in confined structures. Nano Lett. 2010, $10,313-317$.

49. G. A. Beane; K. Gong; D. F. Kelley, Auger and carrier trapping dynamics in core/shell quantum dots having sharp and alloyed interfaces. ACS Nano 2016, 10, 3755-3765.

50. C. She; I. Fedin; D. S. Dolzhnikov; A. Demortière; R. D. Schaller; M. Pelton; D. V. Talapin, Low-threshold stimulated emission using colloidal quantum wells. Nano Lett. 2014, 14, 2772 2777.

51. J. Q. Grim; S. Christodoulou; F. Di Stasio; R. Krahne; R. Cingolani; L. Manna; I. Moreels, Continuous-wave biexciton lasing at room temperature using solution-processed quantum wells. Nat. Nanotechnol. 2014, 9, 891-895.

52. C. She; I. Fedin; D. S. Dolzhnikov; P. D. Dahlberg; G. S. Engel; R. D. Schaller; D. V. Talapin, Red, yellow, green, and blue amplified spontaneous emission and lasing using colloidal CdSe nanoplatelets. ACS Nano 2015, 9, 9475-9485.

53. Z. Yang; M. Pelton; I. Fedin; D. V. Talapin; E. Waks, A room temperature continuous-wave nanolaser using colloidal quantum wells. Nat. Commun. 2017, 8, 143.

54. B. le Feber; F. Prins; E. De Leo; F. T. Rabouw; D. J. Norris, Colloidal-quantum-dot ring lasers with active color control. Nano Lett. 2018, 18, 1028-1034.

55. F. Prins; D. K. Kim; J. Cui; E. De Leo; L. L. Spiegel; K. M. McPeak; D. J. Norris, Direct patterning of colloidal quantum-dot thin films for enhanced and spectrally selective out-coupling of emission. Nano Lett. 2017, 17, 1319-1325.

56. M. P. Hendricks; M. P. Campos; G. T. Cleveland; I. Jen-La Plante; J. S. Owen, A tunable library of substituted thiourea precursors to metal sulfide nanocrystals. Science 2015, 348, 1226-1230.

57. L. R. Doolittle, A semiautomatic algorithm for rutherford backscattering analysis. Nucl. Instrum. Methods Phys. Res. B 1986, 15, 227-231. 

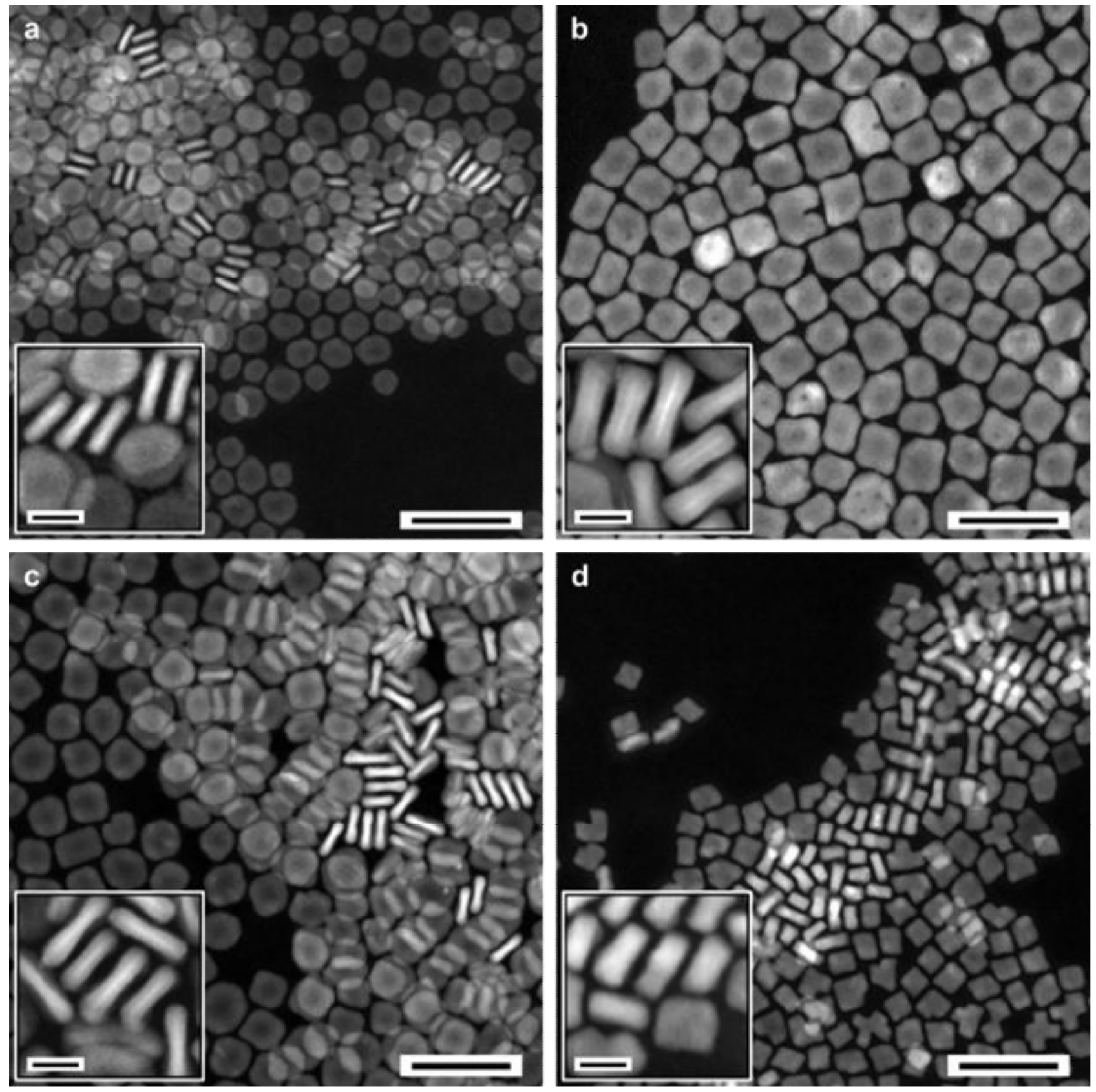

Figure 1. High-angle annular dark-field scanning transmission electron microscopy (HAADF STEM) images of (a) $\mathrm{CdSe} / \mathrm{ZnS}$, (b) $\mathrm{CdSe} / \mathrm{CdS} / \mathrm{ZnS}$, (c) $\mathrm{CdSe} / \mathrm{Cd}_{x} \mathrm{Zn}_{1-\mathrm{x}} \mathrm{S}$, and (d) $\mathrm{CdSe} / \mathrm{ZnSe} \mathrm{NPLs}$. The insets show the corresponding NPLs at higher magnification. Black scale bars are 50 and $10 \mathrm{~nm}$ for the large and inset images, respectively. 


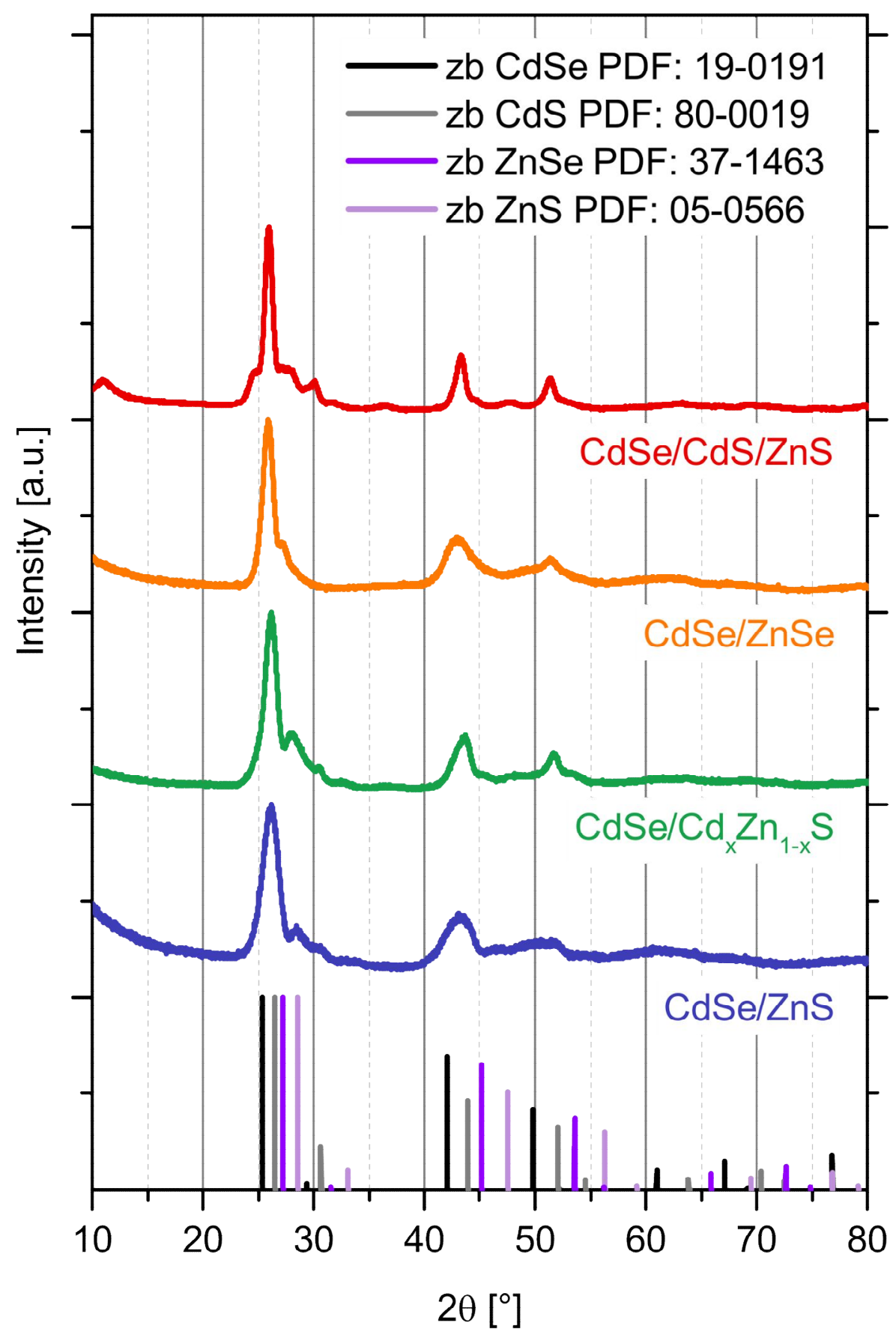

Figure 2. Powder X-ray diffraction (XRD) patterns for the core/shell NPLs shown in Figure 1. Reference patterns (labelled by their powder diffraction file, PDF) are shown for zinc blende crystals of CdSe, CdS, ZnSe, and ZnS. 


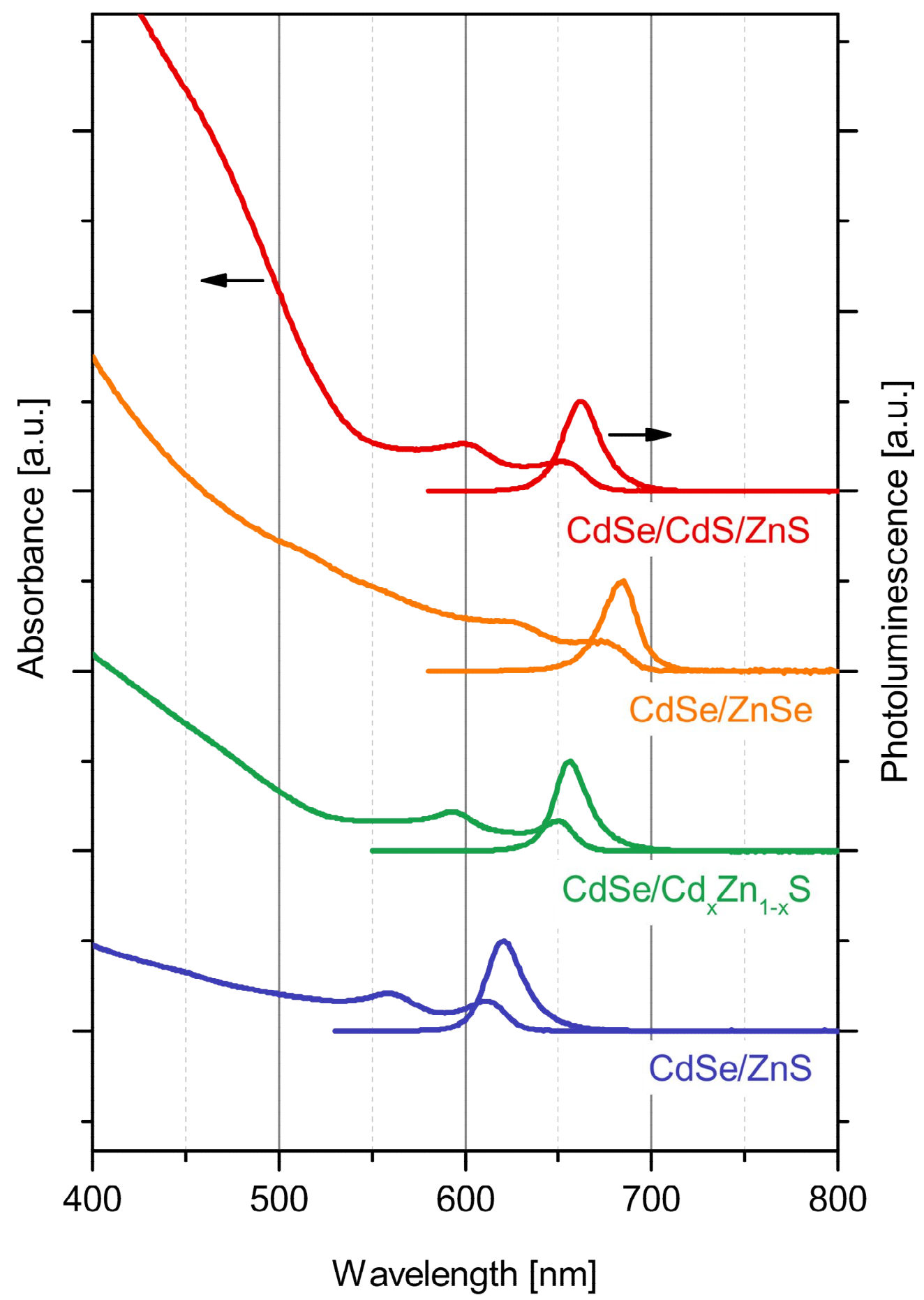

Figure 3. Optical absorption and emission spectra for the core/shell NPLs shown in Figure 1. The emission linewidths range from $56 \mathrm{meV}$ for $\mathrm{CdSe} / \mathrm{Cd}_{\mathrm{x}} \mathrm{Zn}_{1-\mathrm{x}} \mathrm{S}$ to $76 \mathrm{meV}$ for $\mathrm{CdSe} / \mathrm{ZnS}$ core/shell NPLs (see Table S1 in the Supporting Information for all parameters). 

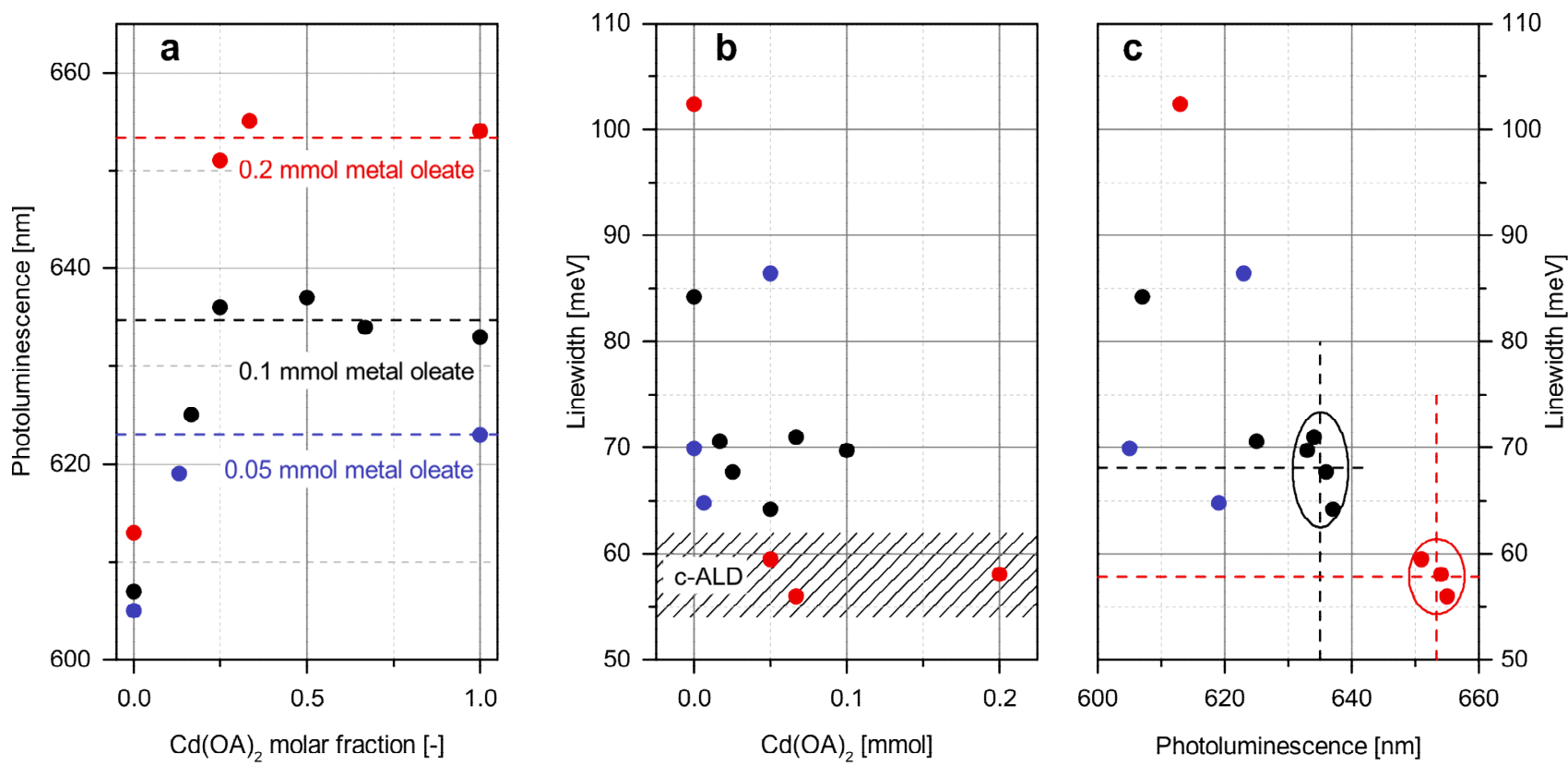

Figure 4. Influence of the precursor ratio and total precursor concentration on the emission properties of $\mathrm{CdSe} / \mathrm{Cd}_{x} \mathrm{Zn}_{1-\mathrm{x}} \mathrm{S}$ NPLs. Blue, black, and red dots correspond to reaction series with $0.05,0.1$, and 0.2 mmols of total metal oleate $\left[\mathrm{Cd}(\mathrm{OA})_{2}+\mathrm{Zn}(\mathrm{OA})_{2}\right]$, respectively. (a) Evolution of the emission wavelength as a function of the $\mathrm{Cd}(\mathrm{OA})_{2}$ molar fraction (relative to the total amount of metal oleate) during the shell growth. (b) Influence of the concentration of $\mathrm{Cd}(\mathrm{OA})_{2}$ on the emission linewidth. The shaded area indicates the linewidth range reported for $\mathrm{CdSe} / \mathrm{CdS}$ core/shell NPLs synthesized by colloidal atomic layer deposition (c-ALD). ${ }^{20-21}$ (c) Relation between the emission wavelength and the corresponding linewidth. The dashed lines indicate the mean photoluminescence wavelengths and linewidths for the reactions using a $\mathrm{Cd}(\mathrm{OA})_{2}$ molar fraction $\geq 25 \%$ (data points inside the circles). 

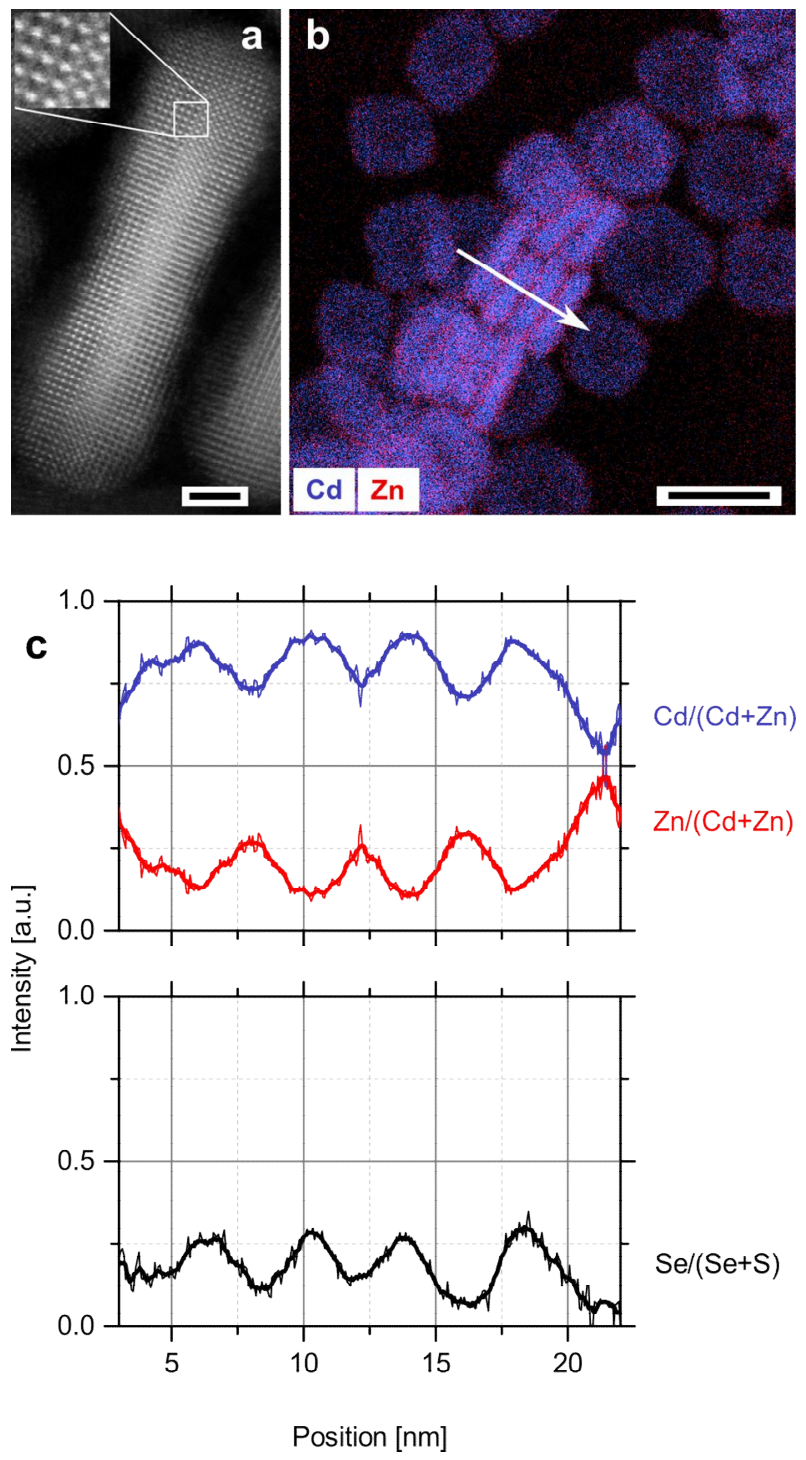

Figure 5. Structural analysis of the $\mathrm{CdSe} / \mathrm{Cd}_{x} \mathrm{Zn}_{1-\mathrm{x}} \mathrm{S}$ core/shell NPLs shown in Figure 1c. (a) Highresolution HAADF STEM cross-sectional image of a single core/shell NPL. The brighter core can be distinguished from the darker shell due to the higher atomic number of the core elements. (b) Superposition of energy-dispersive X-ray spectrographs (EDS) of Cd and Zn for NPLs seen in topand side-view. (c) The corresponding concentration profile across four vertically stacked NPLs, observed from the side. The collection area is marked with the white arrow in (b). Note that a single core/shell NPL in top-view is detected on the left of the stacked NPLs at positions between 0 and $\sim 7 \mathrm{~nm}$ in (c). Black scale bars are $2 \mathrm{~nm}$ in (a) and $20 \mathrm{~nm}$ in (b). 

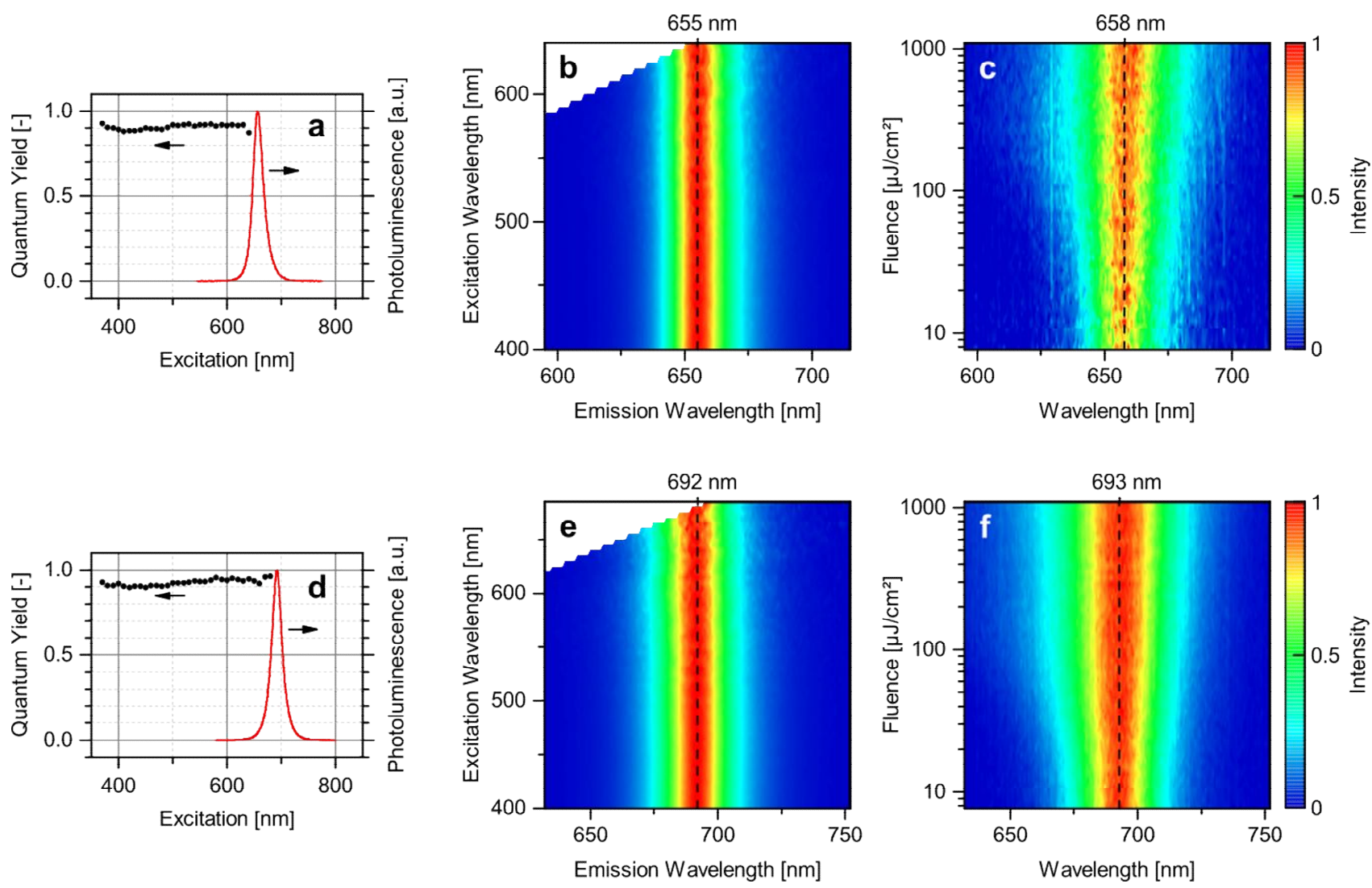

Figure 6. Room-temperature excitation-dependent photoluminescence characterization of $\mathrm{CdSe} / \mathrm{Cd}_{\mathrm{x}} \mathrm{Zn}_{1-\mathrm{x}} \mathrm{S}$ core/shell NPLs having CdSe cores with a thickness of 4 monolayers (a-c) and 6 monolayers (d-f). (a,d) Photoluminescence quantum yields (QYs) measured at different excitation wavelengths. The corresponding emission spectra are shown in red. (b,e) Normalized photoluminescence excitation-emission spectra of core/shell NPL dispersions in hexane. The black vertical dashed lines at $655 \mathrm{~nm}$ (b) and $692 \mathrm{~nm}$ (c) are added for visualization. (c,f) Normalized photoluminescence emission spectra of core/shell NPL films versus the excitation fluence (405 nm excitation, $\sim 340 \mathrm{fs}$ pulse duration, $10 \mathrm{kHz}$ repetition rate). The black vertical dashed lines at $658 \mathrm{~nm}$ (b) and $693 \mathrm{~nm}(\mathrm{c})$ are added for visualization. 

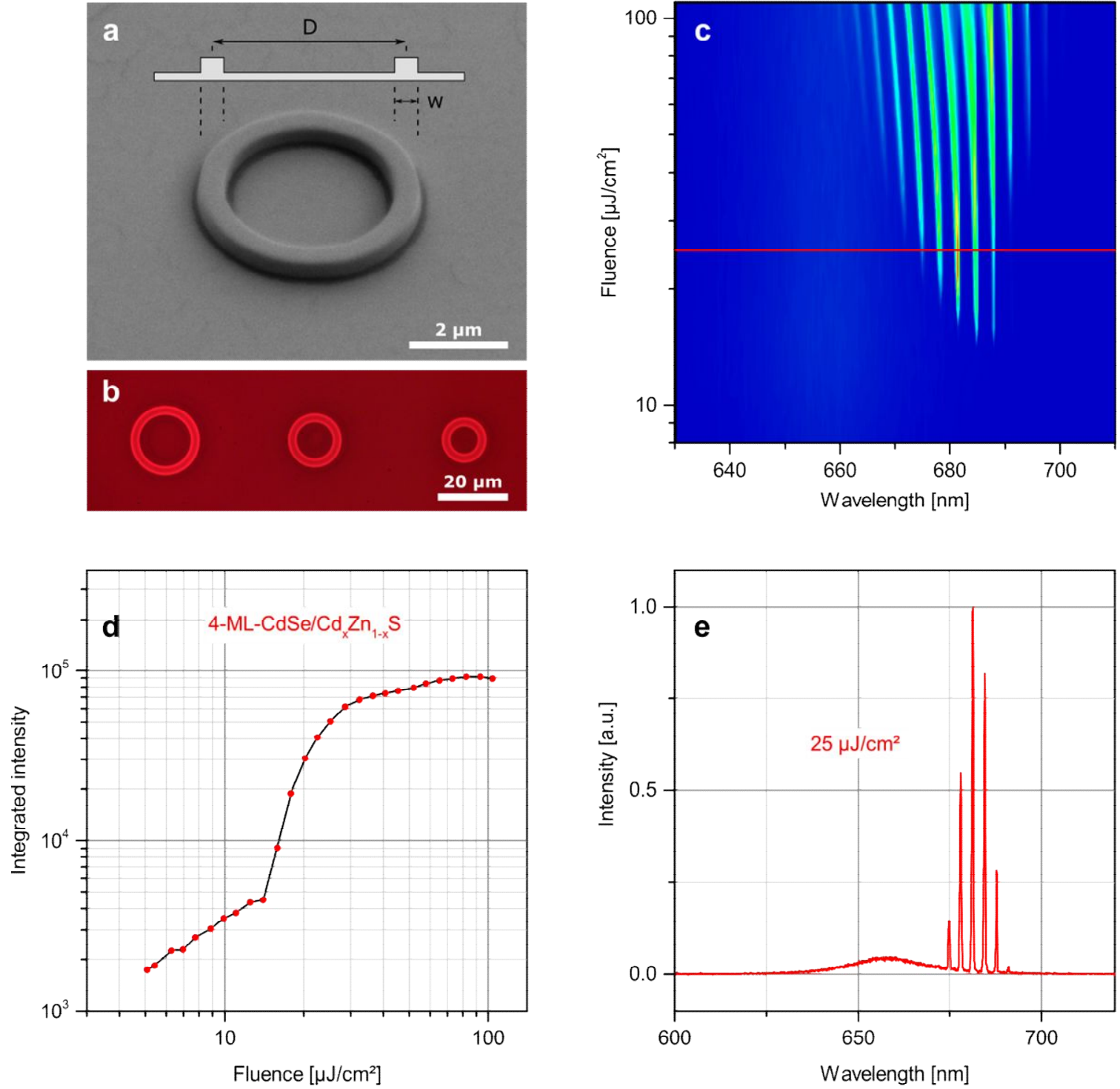

Figure 7. 4-monolayer-CdSe/Cd $\mathrm{Zn}_{1-\mathrm{x}} \mathrm{S}$ core/shell NPL ring resonators and their optical characterization for lasing. (a) Scanning electron micrograph (SEM) of a template-stripped NPL ring resonator. The inset shows a cross-sectional schematic of the ring structure. Rings were fabricated with variable diameters (D) and widths (w) and a height of $\sim 500 \mathrm{~nm}$. (b) Fluorescence image of ring resonators of varying diameters illuminated with ultraviolet light. (c) Power-dependent emission spectra collected from a ring with $\mathrm{D}=20 \mu \mathrm{m}$ and $\mathrm{w}=1 \mu \mathrm{m}$. Narrow lasing lines evolve at fluences above a threshold of $13 \mu \mathrm{J} / \mathrm{cm}^{2}$. (d) Corresponding emission intensity integrated over all collected wavelengths and averaged over the area of the ring resonator versus the excitation fluence. (e) Emission spectrum under lasing conditions extracted at a power of $25 \mu \mathrm{J} / \mathrm{cm}^{2}$ [indicated with a red horizontal line in $(\mathrm{c})]$. 


\title{
Supporting Information
}

\section{High-Temperature Synthesis of CdSe-Based Core/Shell, Core/Shell/Shell, and Core/Graded-Shell Nanoplatelets for Stable and Efficient Narrowband Emitters}

\author{
Aurelio A. Rossinelli, ${ }^{\dagger}$ Henar Rojo,$^{\dagger}$ Aniket S. Mule,$^{\dagger}$ Marianne Aellen $^{\dagger}{ }^{\dagger}$ Ario Cocina ${ }^{\dagger}$ \\ Eva De Leo, ${ }^{\dagger}$ Robin Schäublin, ${ }^{\ddagger}$ and David J. Norris ${ }^{* \dagger}$ \\ ${ }^{\dagger}$ Optical Materials Engineering Laboratory, Department of Mechanical and Process Engineering, \\ ETH Zurich, 8092 Zurich, Switzerland \\ ${ }^{\star}$ Laboratory of Metal Physics and Technology, Department of Materials, \\ ETH Zurich, 8092 Zurich, Switzerland
}

\section{S1. Supporting Experimental Methods}

Optimization of the CdSe/ZnS Core/Shell NPL Morphology. NPLs have a flat and significantly larger surface than QDs. We therefore assumed that the difficulty in obtaining uniform shell morphologies in CdSe/ZnS NPLs is a result of high strain at the core/shell interface. This is supported by the emergence of shape irregularities only for thicker shells (Figure S1). ${ }^{\mathrm{S} 1}$ One approach to minimize interfacial strain is to modify the structure and alloy the interface. With this in mind, we improved the shell morphology by reducing the initial precursor concentration and increasing the monomer solubility. We added only $25 \%$ of the total amount of $\mathrm{Zn}(\mathrm{OA})_{2}$ at the beginning and injected the remaining part along with 1-octanethiol at $300{ }^{\circ} \mathrm{C}$. Furthermore, we injected oleic acid (OA) together with 1-octanethiol. This excess of OA was necessary to avoid the formation of irregularly shaped shells (Figure S2). It is well known that $\mathrm{OA}$ increases the monomer solubility and reduces the growth rate. ${ }^{\mathrm{S} 2}$ The core/shell 
structure was then analyzed by HAADF STEM and EDS (Figure 1a in the main text and Figure S4). We measured a shell thickness of $1-1.5 \mathrm{~nm}$, which is smaller than for CdSe/CdS NPLs using the same amount of precursor. The EDS analysis further revealed the presence of Cd and Se in both the core as well as the inner part of the shell. Partly, this can be explained by diffusion. At such high reaction temperatures cation diffusion is enhanced, ${ }^{\mathrm{S} 3}$ which can lead to alloying of $\mathrm{Cd}$ and $\mathrm{Zn}$ at the core/shell interface. In contrast, anion diffusion is substantially slower, and we do not observe strong anion diffusion into the shell of CdSe/CdS NPLs synthesized under the same conditions. However, a reduction of the lateral size of $\mathrm{CdSe} / \mathrm{ZnS}$ NPLs compared to CdSe/CdS NPLs can be observed. We believe that the smaller lateral size results from a prolonged initial etching of the CdSe NPLs due to the slower growth rate. The subsequent incorporation of this dissolved material into the shell enhances interfacial alloying and explains the detection of $\mathrm{Cd}$ and Se in the shell.

\section{S2. Supporting Tables}

\begin{tabular}{lccccc}
\hline Material & $\begin{array}{c}\text { Emission Peak } \\
{[\mathbf{n m}]}\end{array}$ & $\begin{array}{c}\text { FWHM } \\
{[\mathbf{m e V}]}\end{array}$ & $\begin{array}{c}\text { FWHM } \\
{[\mathbf{n m}]}\end{array}$ & $\begin{array}{c}\text { QY } \\
{[\%]}\end{array}$ & $\begin{array}{c}\boldsymbol{\tau}_{\text {avg }} \\
{[\mathbf{n s}]}\end{array}$ \\
\hline 4-monolayer-CdSe/Cd $\mathrm{Zn}_{1-\mathrm{x}} \mathrm{S}$ & 655 & 56 & 19.5 & 88 & 25 \\
6-monolayer-CdSe/Cd $\mathrm{Zn}_{1-\mathrm{x}} \mathrm{S}$ & 692 & 57 & 22.1 & 92 & 22 \\
$\mathrm{CdSe} / \mathrm{CdS} / \mathrm{ZnS}$ & 662 & 67 & 23.6 & 78 & 37 \\
$\mathrm{CdSe} / \mathrm{ZnS}$ (uniform) & 620 & 76 & 23.7 & 52 & 10 \\
$\mathrm{CdSe} / \mathrm{ZnS}$ (rough) & 612 & 101 & 30.7 & 67 & 21 \\
$\mathrm{CdSe} / \mathrm{ZnSe}$ & 684 & 59 & 22.1 & 47 & 13 \\
\hline
\end{tabular}

$\tau_{\text {avg: }}$ average lifetime

Table S1. Summary of the room-temperature optical properties for the core/shell NPLs presented in Figures 1-7 in the main text. The columns describe the peak wavelength of the emission, its full-width-at-half-maximum (FWHM) in $\mathrm{meV}$ and $\mathrm{nm}$, respectively, photoluminescence quantum yield (QY), and average photoluminescence decay time, $\tau_{\text {avg. }}$. 


\section{S3. Supporting Figures}
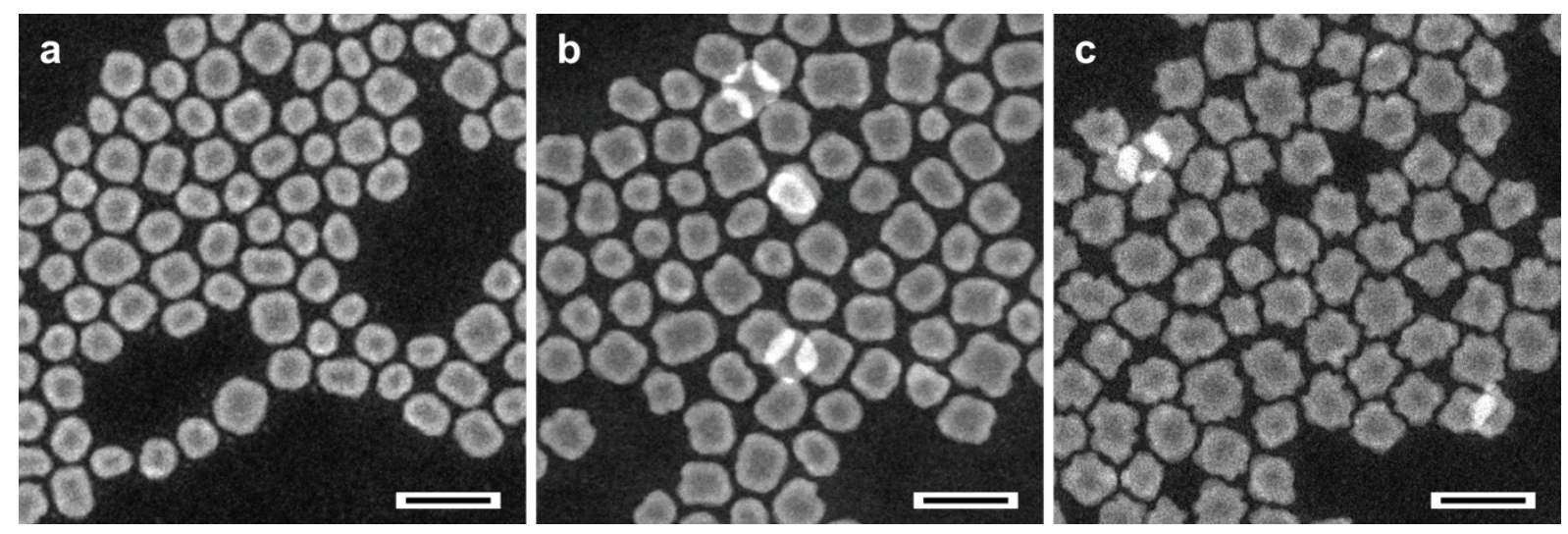

Figure S1. Influence of the ZnS shell thickness on the shell morphology of CdSe/ZnS NPLs.

(a) Thin $\mathrm{ZnS}$ shell using a small initial concentration $(0.05 \mathrm{mmol})$ of $\mathrm{Zn}(\mathrm{OA})_{2}$. (b) Intermediate $\mathrm{ZnS}$ shell thickness using $0.1 \mathrm{mmol}$ of $\mathrm{Zn}(\mathrm{OA})_{2}$. (c) Thickest $\mathrm{ZnS}$ shell using $0.2 \mathrm{mmol}$ $\mathrm{Zn}(\mathrm{OA})_{2}$. The reactions were performed as described in the paragraph labelled "Optimization of CdSe/Cd $\mathrm{Zn}_{1-\mathrm{x}} \mathrm{S}$ Core/Shell NPLs" in the Experimental Section of the main text. Scale bars are $30 \mathrm{~nm}$. 

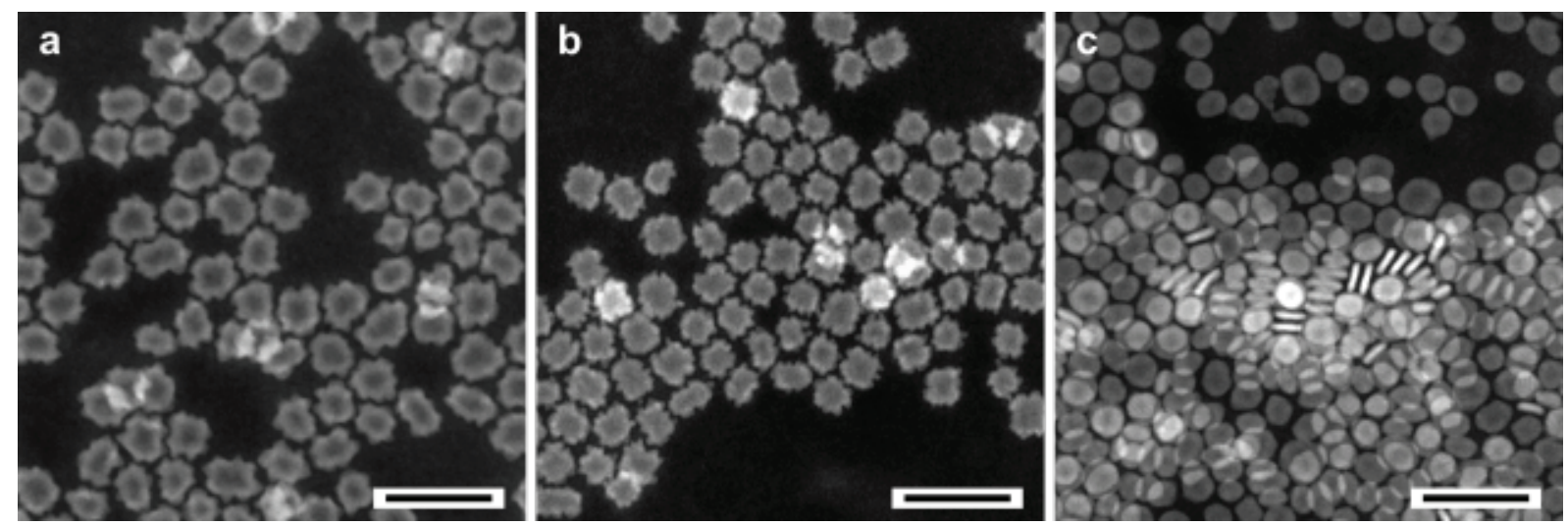

Figure S2. Influence of the synthesis conditions on the shell morphology of CdSe/ZnS NPLs.

(a) High initial concentration $(0.2 \mathrm{mmol})$ of $\mathrm{Zn}(\mathrm{OA})_{2}$ and no addition of oleic acid (OA) during the injection of 1-octanethiol. (b) Reduced initial concentration of $\mathrm{Zn}(\mathrm{OA})_{2}(0.05 \mathrm{mmol})$ and no addition of OA during the injection of 1-octanethiol. (c) Reduced initial concentration of $\mathrm{Zn}(\mathrm{OA})_{2}(0.05 \mathrm{mmol})$ and addition of OA during the injection of 1-octanethiol. Black scale bars are $50 \mathrm{~nm}$.

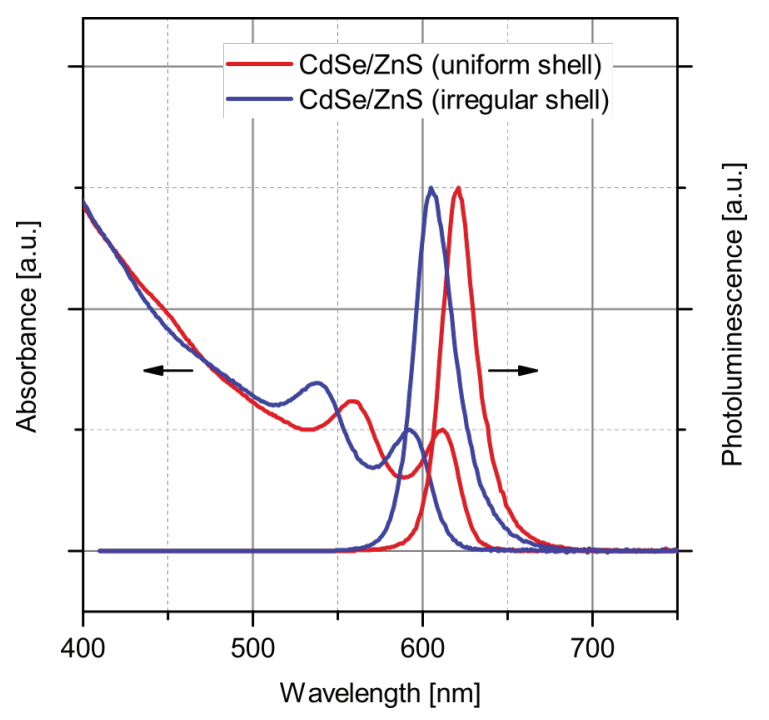

Figure S3. Influence of the shell morphology on the absorption and emission spectra of CdSe/ZnS NPLs. NPLs with a uniform shell (as determined by electron microscopy) show reduced emission linewidths and red-shifted emission. 

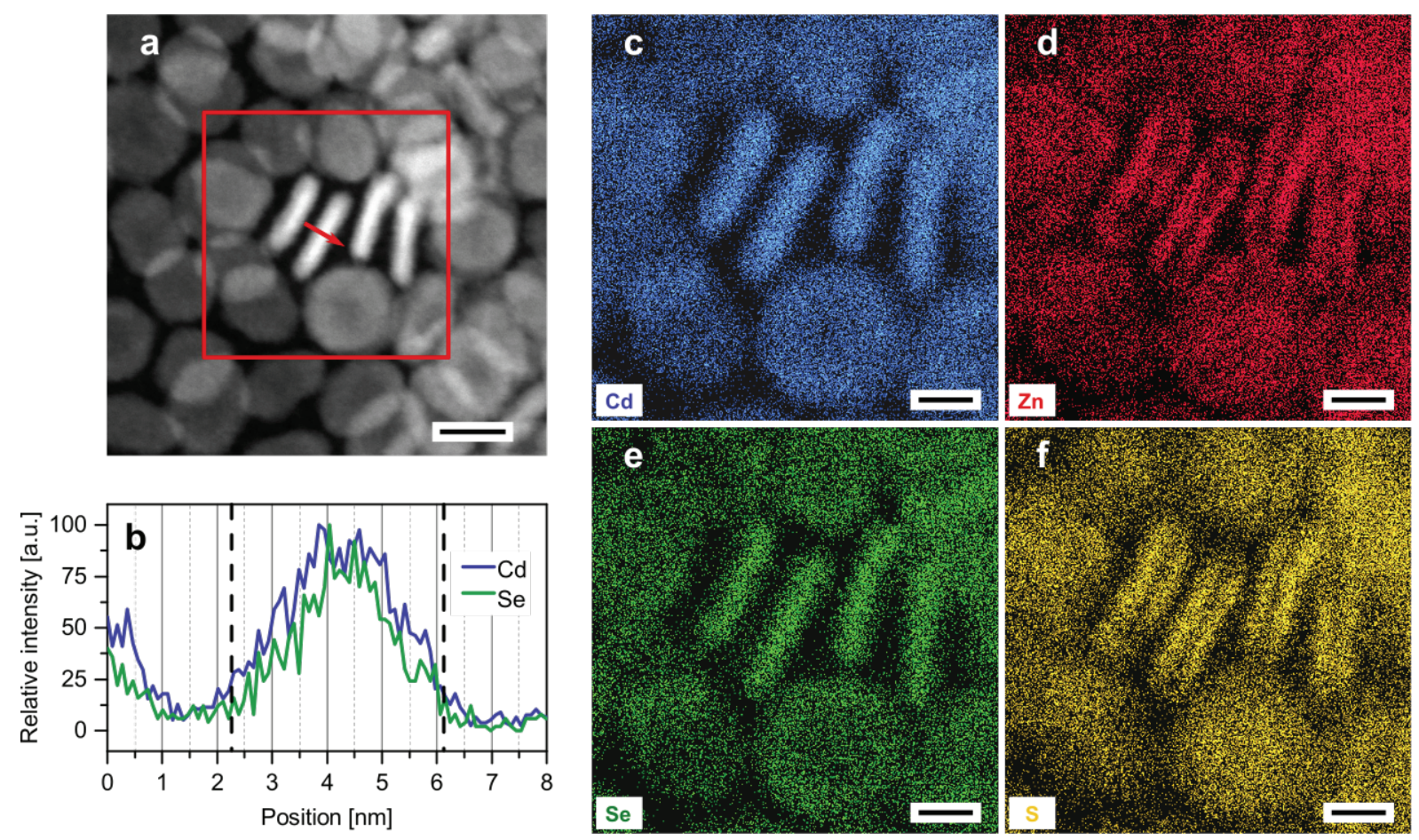

Figure S4. Structural analysis of CdSe/ZnS core/shell NPLs with a uniform shell. (a) HAADF STEM image. (b) Cd and Se concentration profile across a single CdSe/ZnS NPL. The profile is measured on the NPL marked with a red arrow in (a). Dashed lines indicate the width of the NPL. (c)-(f) Energy dispersive X-ray spectroscopy (EDS) images for Cd, Zn, Se, and S. Scale bars are $10 \mathrm{~nm}$ in (a) and $5 \mathrm{~nm}$ in (c)-(f).
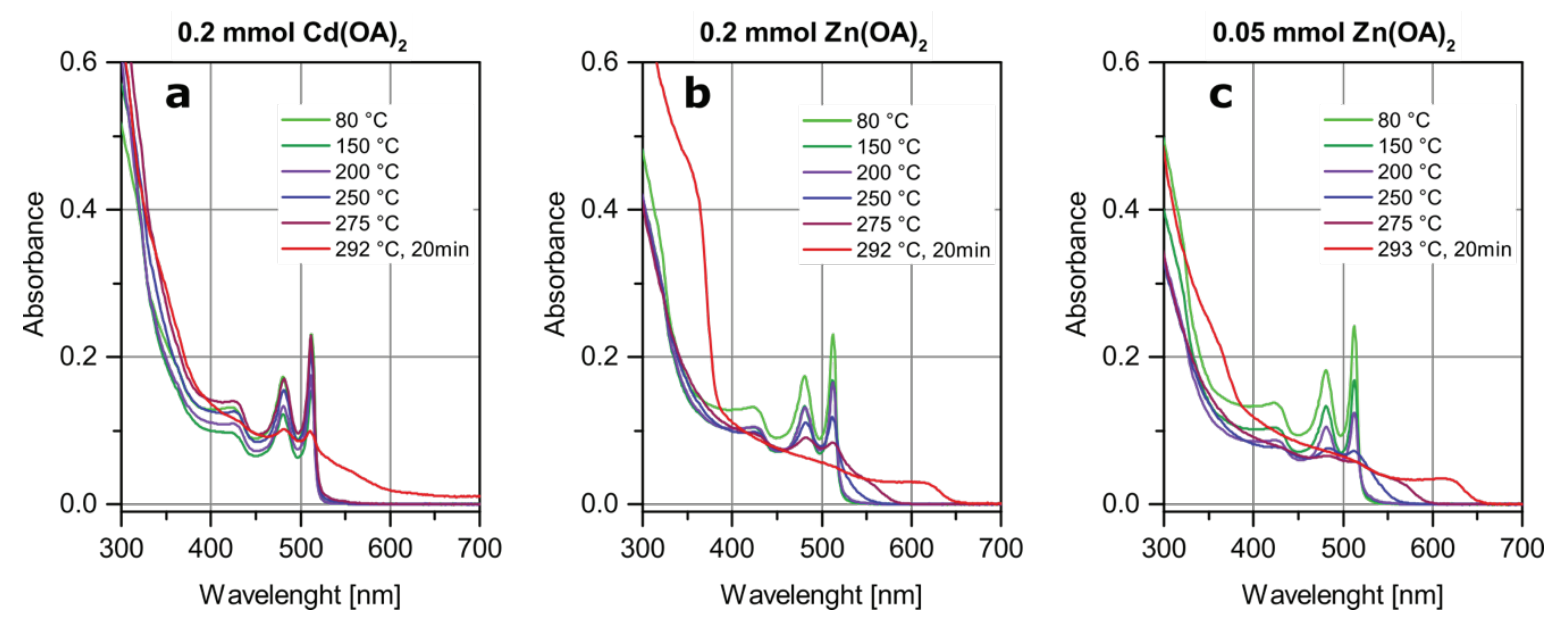

Figure S5. Temporal evolution of the NPL absorption features during heating of the CdSe NPLs dispersed in ODE + OA + OAm with either (a) $0.2 \mathrm{mmol} \mathrm{Cd(OA})_{2}$, (b) $0.2 \mathrm{mmol}$ $\mathrm{Zn}(\mathrm{OA})_{2}$, or (c) $0.05 \mathrm{mmol} \mathrm{Zn}(\mathrm{OA})_{2}$. The first data points, denoted as " $80{ }^{\circ} \mathrm{C}$ ", were taken immediately after the addition of OAm at $80{ }^{\circ} \mathrm{C}$. The last data points were taken after $20 \mathrm{~min}$. 
$0.2 \mathrm{mmol} \mathrm{Cd}(\mathrm{OA})_{2}$

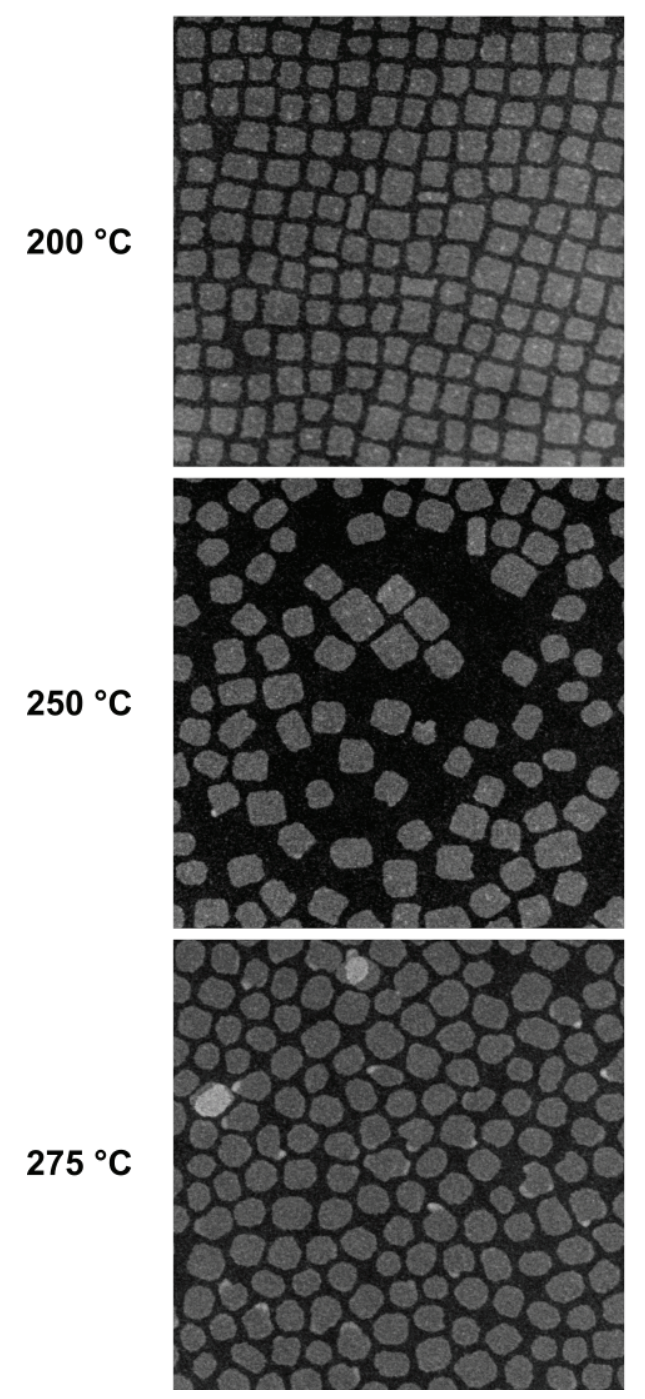

$0.2 \mathrm{mmol} \mathrm{Zn}(\mathrm{OA})_{2}$
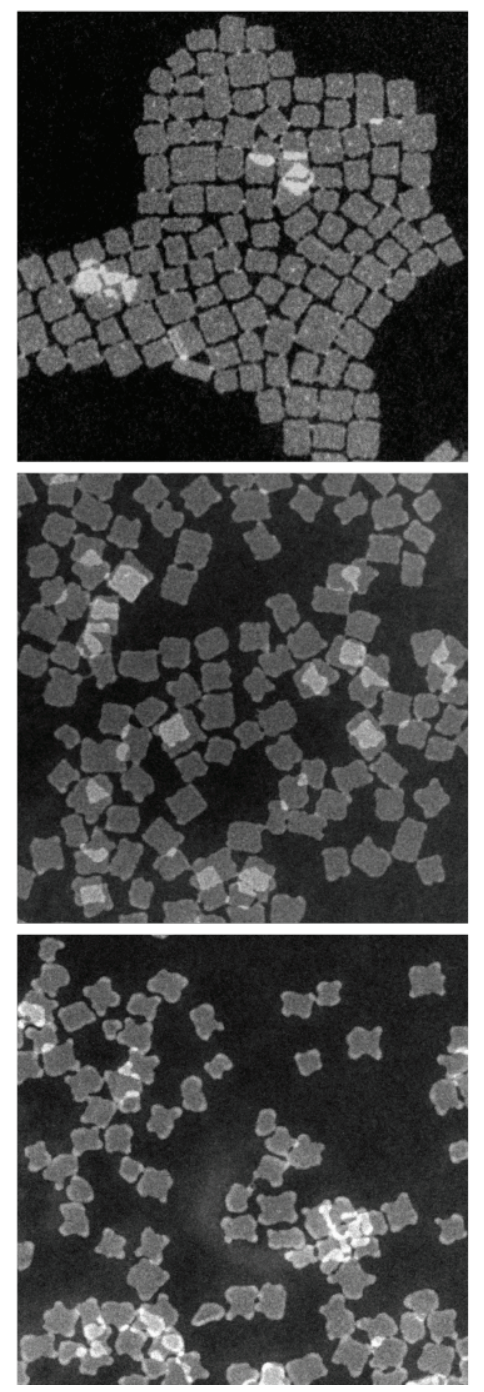

$0.05 \mathrm{mmol} \mathrm{Zn}(\mathrm{OA})_{2}$
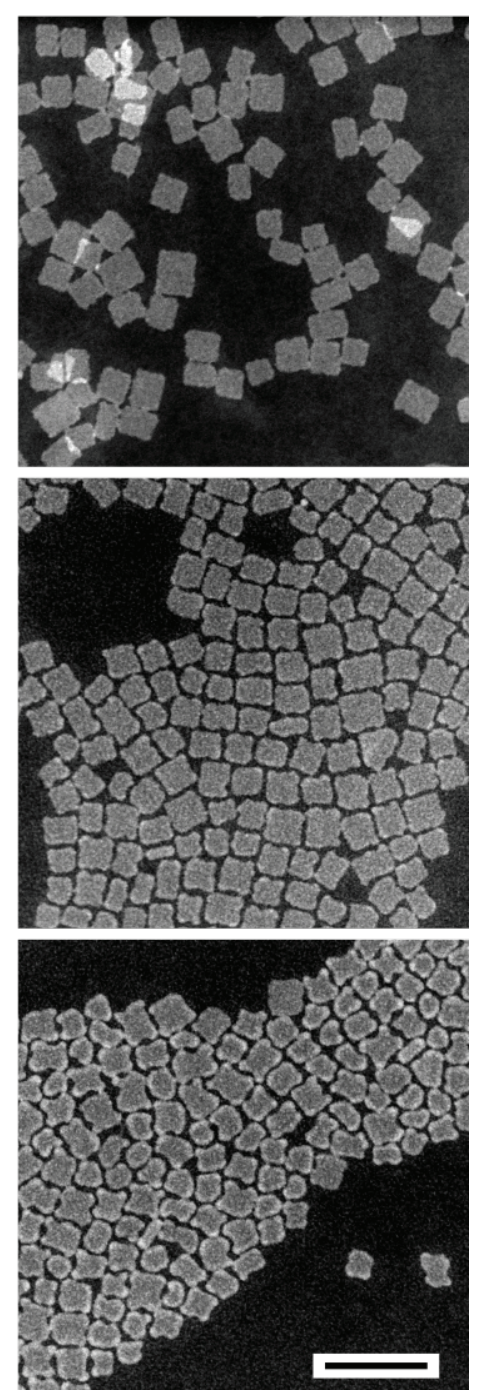

Figure S6. HAADF STEM images showing the temporal evolution of the NPL geometry during heating of the CdSe NPLs dispersed in ODE + OA + OAm with either $0.2 \mathrm{mmol} \mathrm{Cd}(\mathrm{OA})_{2}$, $0.2 \mathrm{mmol} \mathrm{Zn}(\mathrm{OA})_{2}$, or $0.05 \mathrm{mmol} \mathrm{Zn}(\mathrm{OA})_{2}$. The samples are taken at temperatures of $200{ }^{\circ} \mathrm{C}$, $250{ }^{\circ} \mathrm{C}$, and $275^{\circ} \mathrm{C}$. Black scale bar is $50 \mathrm{~nm}$ (all STEM images are at the same magnification). 

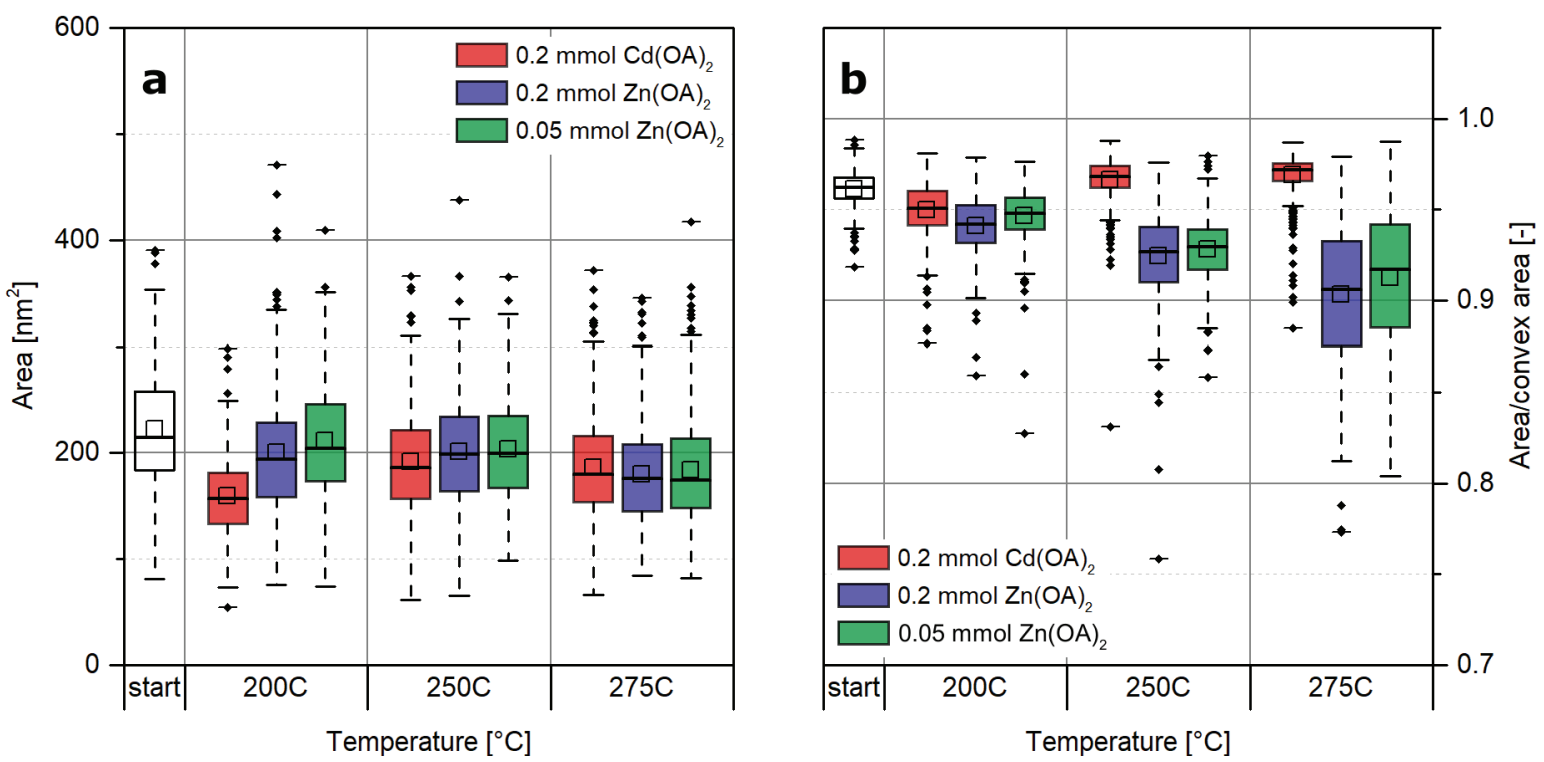

Figure S7. Statistical analysis of the temporal evolution of the size and shape of CdSe NPLs recorded by HAADF STEM (Figure S6). (a) Evolution of the cross-sectional area. (b) Crosssectional area normalized by the convex hull area, describing the uniformity of the CdSe NPLs. Box plots show a rectangle representing the interquartile range (IQR) and whiskers at $1.5 \mathrm{x}$ IQR. The median (horizontal line within the rectangle), mean (open square within the box), and outliers (filled diamonds) are also shown. Statistics were computed from 400 NPLs for each aliquot. 

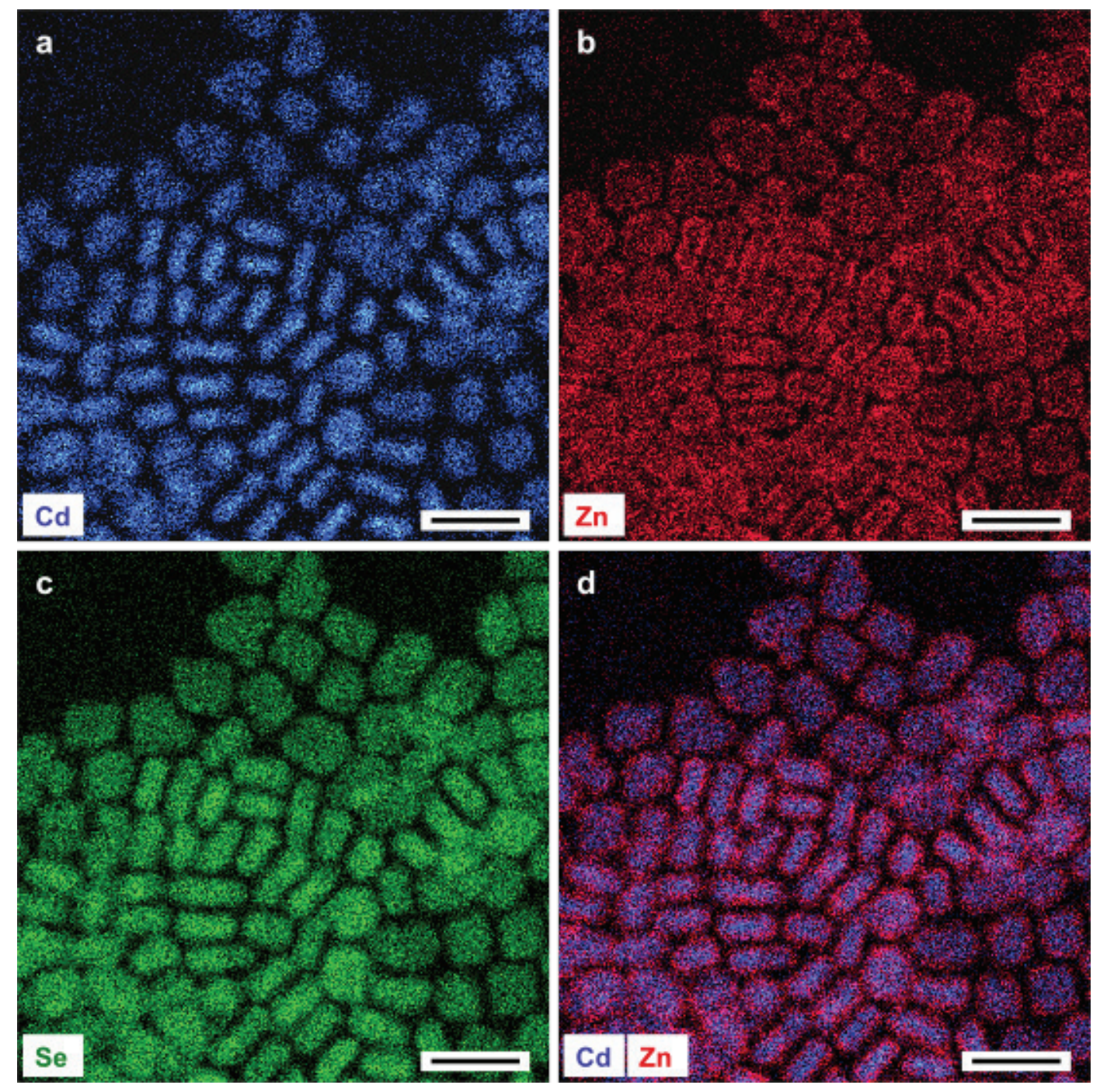

Figure S8. Compositional analysis of CdSe/ZnSe core/shell NPLs. (a)-(c) Energy dispersive X-ray spectroscopy (EDS) images of Cd, Zn, Se, respectively, and (d) the superposition of Cd and $\mathrm{Zn}$. Black scale bars are $20 \mathrm{~nm}$. 


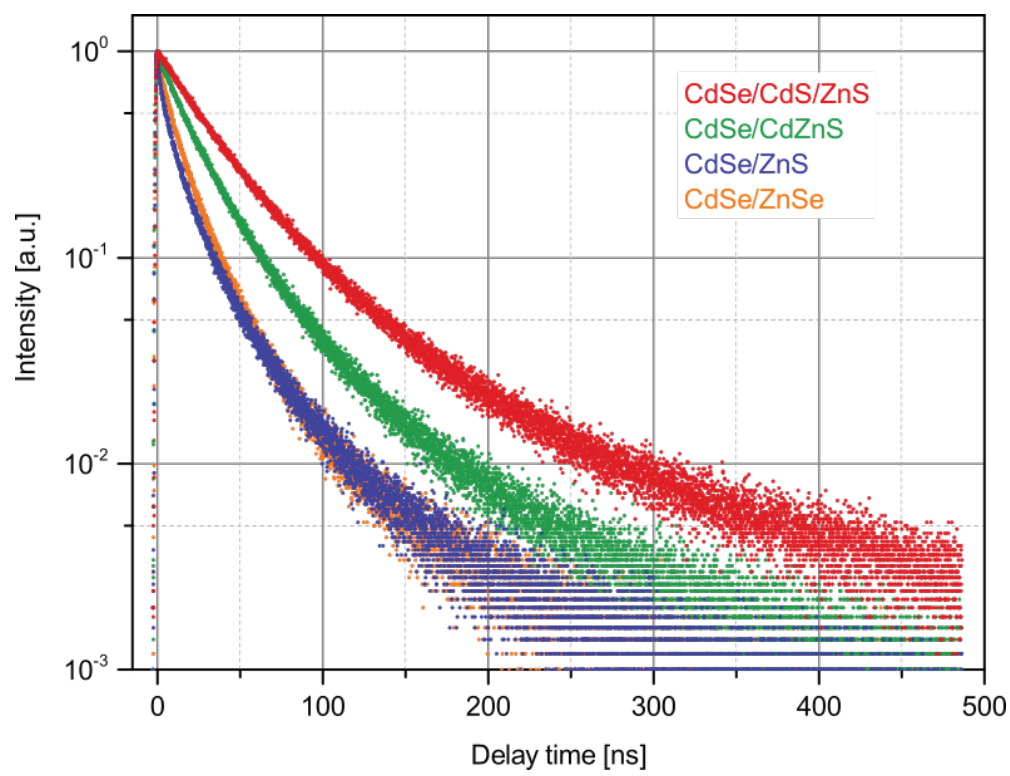

Figure S9. Room-temperature photoluminescence decays of $\mathrm{CdSe} / \mathrm{CdS} / \mathrm{ZnS}, \mathrm{CdSe} / \mathrm{Cd}_{\mathrm{x}} \mathrm{Zn}_{1-\mathrm{x}} \mathrm{S}$, $\mathrm{CdSe} / \mathrm{ZnS}$, and $\mathrm{CdSe} / \mathrm{ZnSe}$ NPLs dispersed in hexane. The detected wavelengths were set to the emission maxima of the respective NPLs. Due to the larger delocalization of the electronic wave function into the shell and the higher QYs, the radiative lifetimes are expected to be longer in the $\mathrm{CdSe} / \mathrm{CdS} / \mathrm{ZnS}$ and $\mathrm{CdSe} / \mathrm{Cd}_{\mathrm{x}} \mathrm{Zn}_{1-\mathrm{x}} \mathrm{S}$ NPLs. 

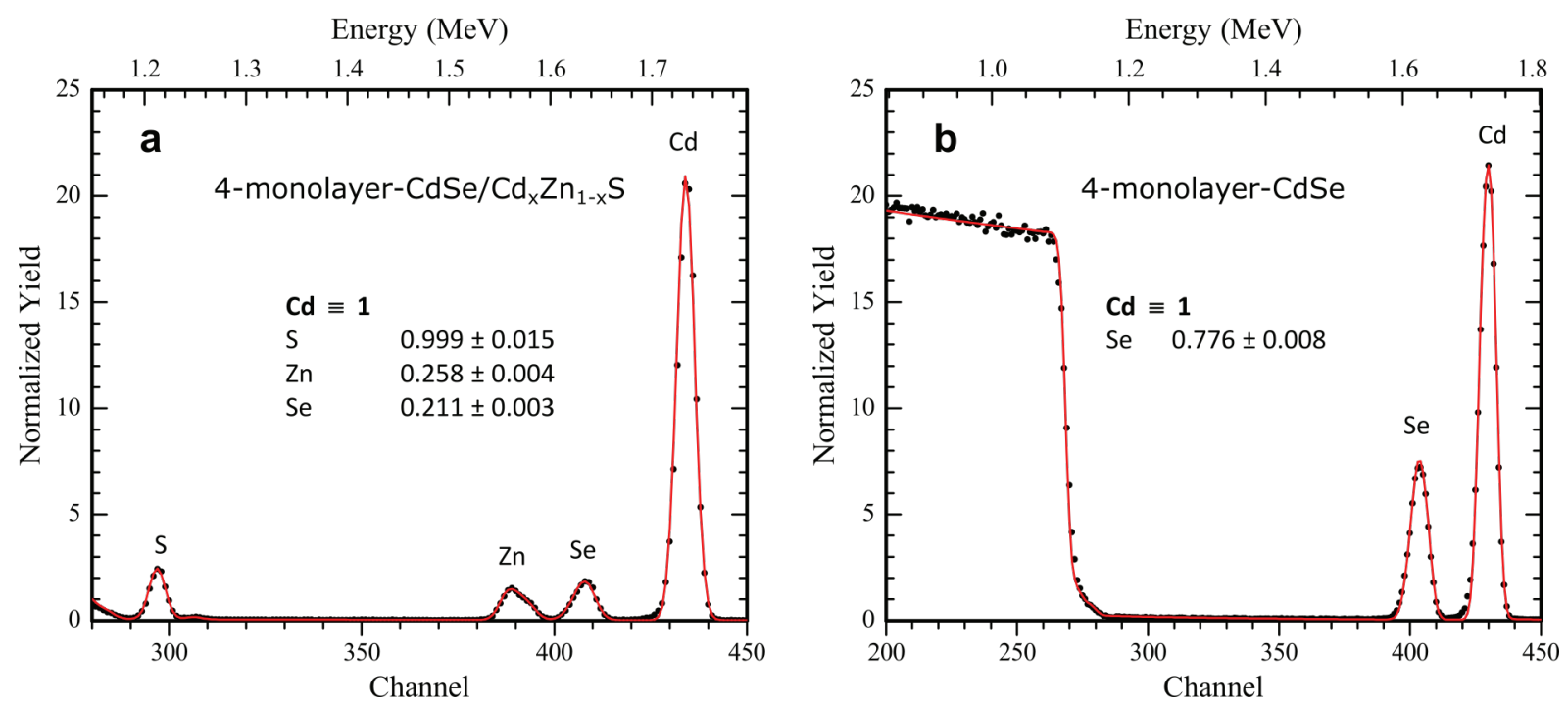

Figure S10. Rutherford back scattering (RBS) spectra of (a) 4-monolayer-CdSe/Cd $\mathrm{Zn}_{1-\mathrm{x}} \mathrm{S}$ NPLs synthesized using a precursor ratio $\left[\mathrm{Cd}(\mathrm{OA})_{2}\right]:\left[\mathrm{Zn}(\mathrm{OA})_{2}\right]$ of $1: 2$ and $(\mathrm{b})$ 4-monolayerCdSe NPLs. Elemental concentrations are normalized with respect to [Cd] and show an excess of cations $(\mathrm{Cd}, \mathrm{Zn})$ for both core and core/shell NPLs, indicating a metal-rich surface.
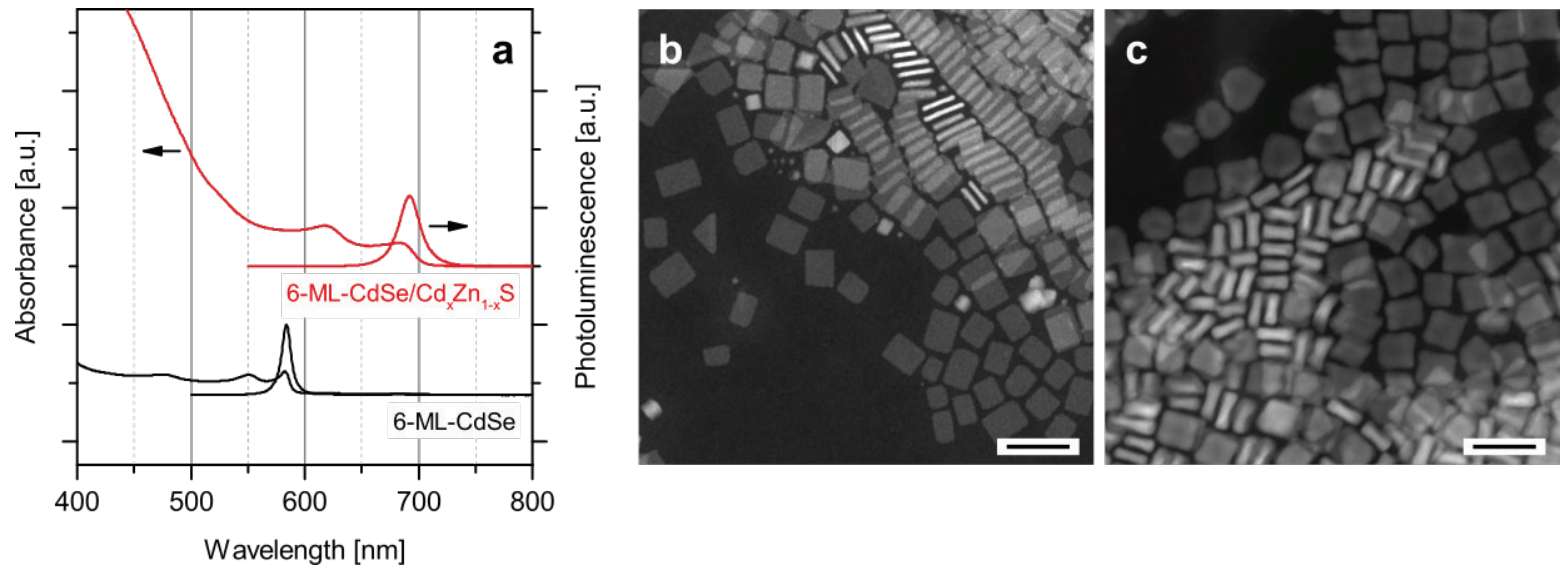

Figure S11. (a) Absorption and emission spectra of 6-monolayer-thick CdSe NPLs (black) and 6-monolayer-CdSe/ $\mathrm{Cd}_{\mathrm{x}} \mathrm{Zn}_{1-\mathrm{x}} \mathrm{S}$ NPLs synthesized using a precursor ratio $\left[\mathrm{Cd}(\mathrm{OA})_{2}\right]:\left[\mathrm{Zn}(\mathrm{OA})_{2}\right]$ of 1:2 (red). (b, c) Corresponding HAADF STEM images of the CdSe core and core/shell NPLs. Black scale bars are $30 \mathrm{~nm}$. 

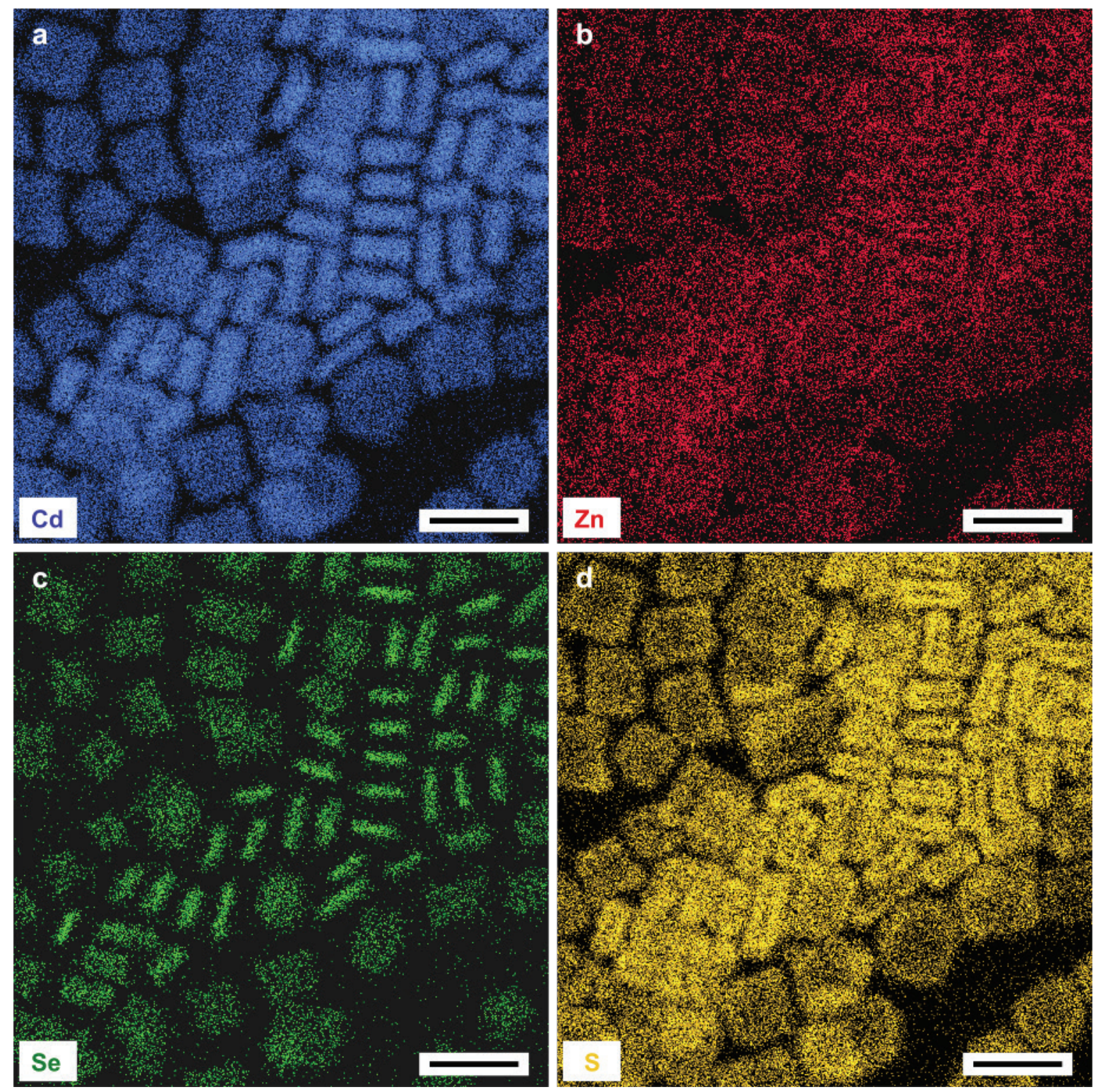

Figure S12. Energy dispersive X-ray spectroscopy (EDS) images of $\mathrm{Cd}, \mathrm{Zn}, \mathrm{Se}$, and $\mathrm{S}$ of $\mathrm{CdSe} / \mathrm{Cd}_{\mathrm{x}} \mathrm{Zn}_{1-\mathrm{x}} \mathrm{S}$ NPLs using 6-monolayer-thick CdSe NPL cores. Due to the thicker geometry, these core CdSe NPLs show an improved thermal stability and the original rectangular shape is largely preserved in the final core/shell NPL structures. Black scale bars are $20 \mathrm{~nm}$. 

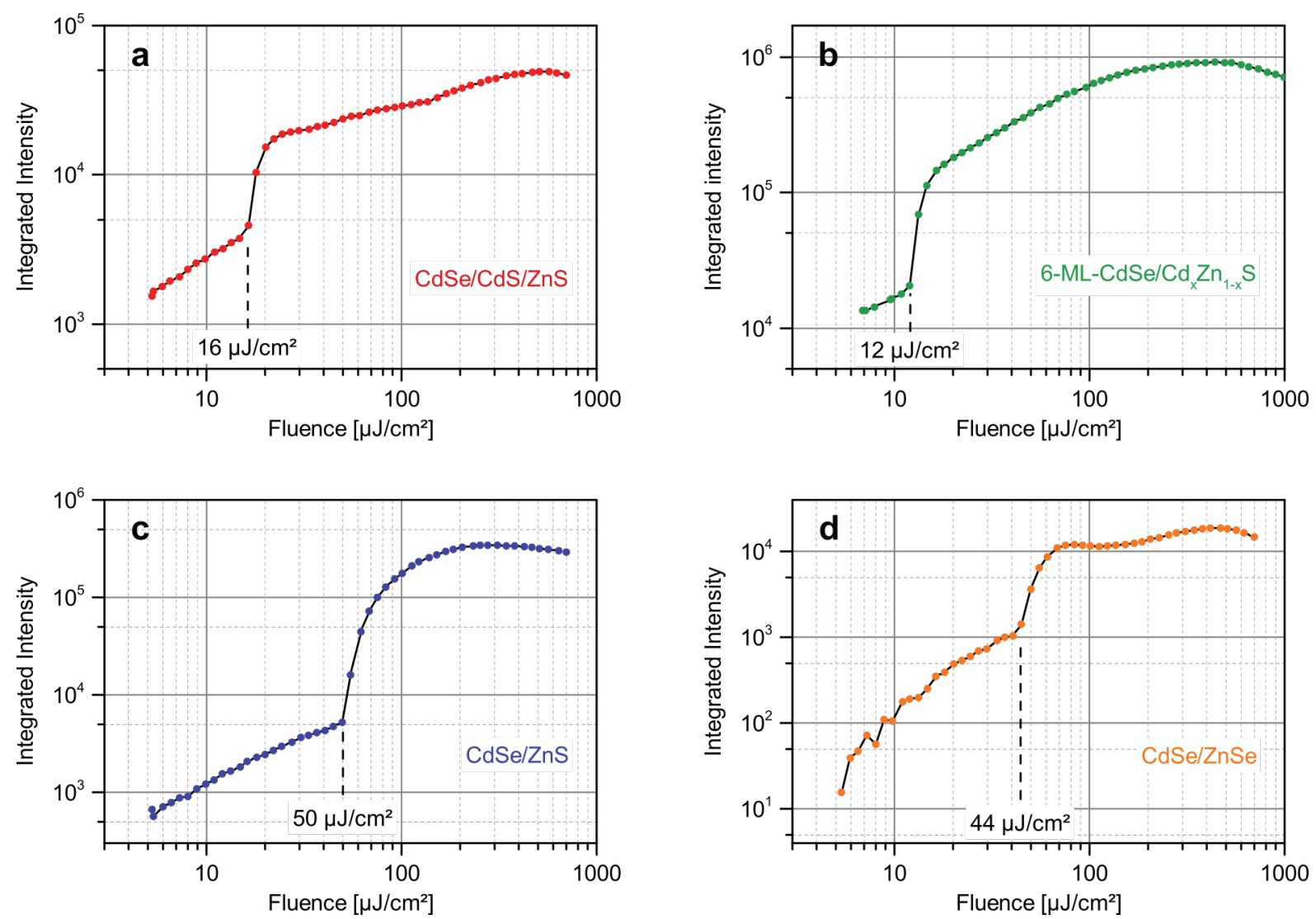

Figure S13. Power-dependent emission intensity versus the excitation fluence, measured for (a) $\mathrm{CdSe} / \mathrm{CdS} / \mathrm{ZnS}$, (b) $\mathrm{CdSe} / \mathrm{Cd}_{\mathrm{x}} \mathrm{Zn}_{1-\mathrm{x}} \mathrm{S}$, (c) $\mathrm{CdSe} / \mathrm{ZnS}$, and (d) $\mathrm{CdSe} / \mathrm{ZnSe}$ core/shell NPL ring lasers with ring diameter $\mathrm{D}=20 \mu \mathrm{m}$ and width $\mathrm{w}=1 \mu \mathrm{m}$ (see Figure $7 \mathrm{a}$ in the main text for a definition of these parameters). The emission was integrated over all collected wavelengths and averaged over the ring area. All core/shell NPLs show low lasing thresholds below a fluence of $50 \mu \mathrm{m} / \mathrm{cm}^{2}$. 

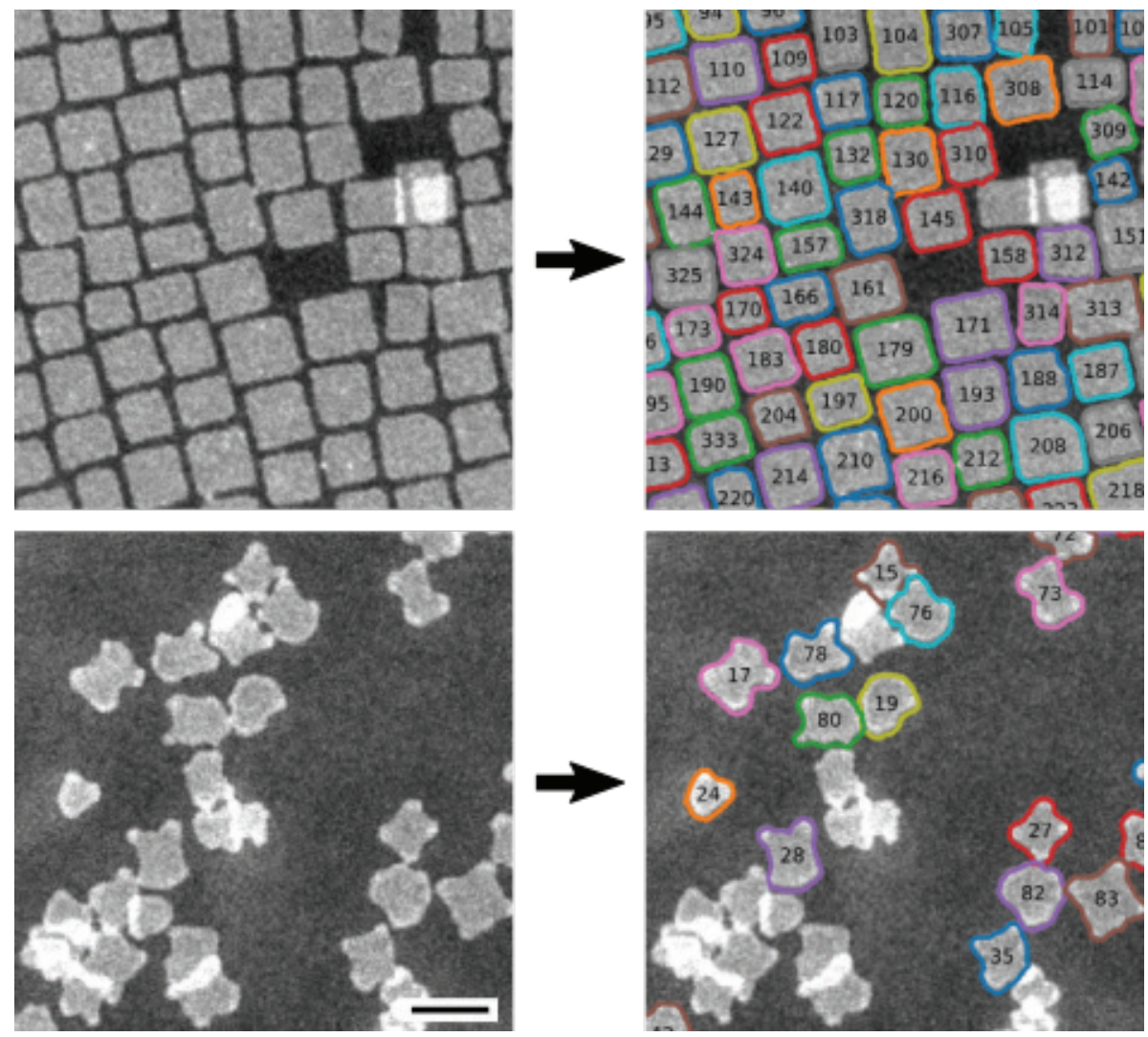

Figure S14. Visualization of the NPL size and shape evolution. On the left, two sections of raw HAADF STEM images that were analyzed are displayed. On the right, the contour of the detected NPLs is superimposed with the original image. NPLs that overlay each other are removed from the analysis. For more details on the analysis method see the Experimental Section in the main text. The black scale bar is $50 \mathrm{~nm}$. All images are at the same magnification. 


\section{S4. Supporting References}

S1. S. G. Kwon; G. Krylova; P. J. Phillips; R. F. Klie; S. Chattopadhyay; T. Shibata; E. E. Bunel; Y. Liu; V. B. Prakapenka; B. Lee; E. V. Shevchenko, Heterogeneous nucleation and shape transformation of multicomponent metallic nanostructures. Nat. Mater. 2015, $14,215-223$.

S2. S. Abe; R. K. Capek; B. De Geyter; Z. Hens, Reaction chemistry/nanocrystal property relations in the hot injection synthesis, the role of the solute solubility. ACS Nano 2013, 7, 943-949.

S3. D. Shaw, Diffusion mechanisms in II-VI materials. J. Cryst. Growth 1988, 86, 778-796. 
